This item was submitted to Loughborough's Research Repository by the author.

Items in Figshare are protected by copyright, with all rights reserved, unless otherwise indicated.

\title{
An analytical approach for analysis and optimisation of slider bearings with infinite width parallel textures
}

PLEASE CITE THE PUBLISHED VERSION

http://dx.doi.org/10.1016/j.triboint.2010.02.016

PUBLISHER

(C) Elsevier

VERSION

AM (Accepted Manuscript)

LICENCE

CC BY-NC-ND 4.0

\section{REPOSITORY RECORD}

Rahmani, Ramin, Iraj Mirzaee, Ayoub Shirvani, and Hassan Shirvani. 2019. "An Analytical Approach for Analysis and Optimisation of Slider Bearings with Infinite Width Parallel Textures". figshare.

https://hdl.handle.net/2134/12903. 
This item was submitted to Loughborough's Institutional Repository (https://dspace.lboro.ac.uk/) by the author and is made available under the following Creative Commons Licence conditions.

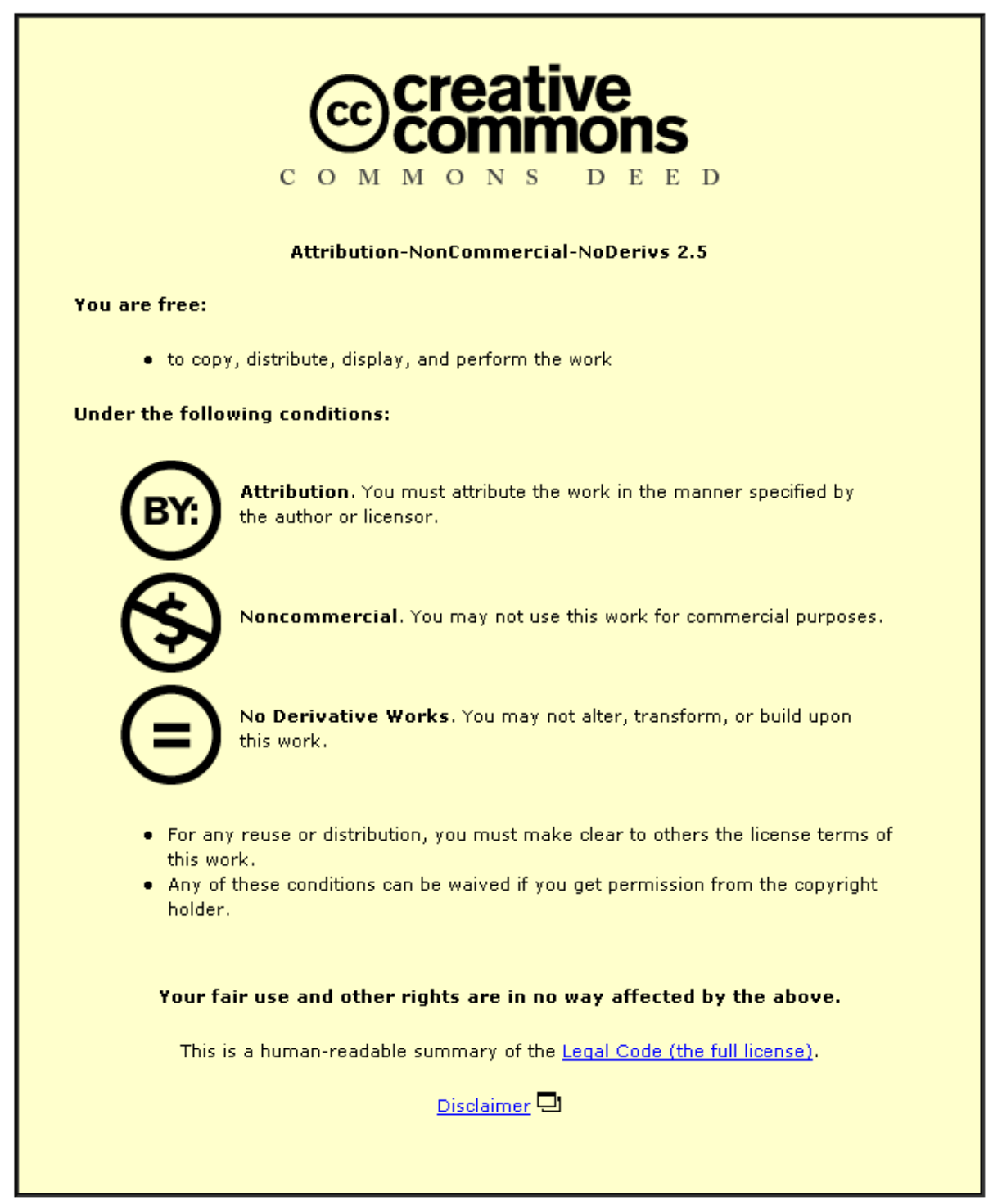

For the full text of this licence, please go to: http://creativecommons.org/licenses/by-nc-nd/2.5/ 


\title{
An Analytical Approach for Analysis and Optimisation of Slider Bearings with Infinite Width Parallel Textures
}

\author{
R. Rahmani ${ }^{\mathrm{a}_{1}}$, I. Mirzaee ${ }^{\mathrm{b}}$, A. Shirvani ${ }^{\mathrm{C} 3}, \mathrm{H}$. Shirvani ${ }^{\mathrm{C} 4}$ \\ ${ }^{a}$ Wolfson School of Mechanical and Manufacturing Engineering, Loughborough \\ University, Loughborough, Leicestershire, LE11 3TU, UK \\ Phone: +44 (0) 150922 7686, Fax: +44 (0) 1245227648 \\ Email: $\underline{\text { r.rahmani@lboro.ac.uk }}$ \\ ${ }^{\mathrm{b}}$ Urmia University of Technology, Band Highway, Urmia, Iran \\ Phone: +98 (0) 441 3554183, Fax: +98 (0) 4413554184 \\ ${ }^{\mathrm{c}}$ Department of Computing Science, Faculty of Science and Technology, Anglia \\ Ruskin University, Bishop Hall Lane, Chelmsford, Essex CM1 1SQ, UK \\ Phone: +44 (0) 1245 493131, Fax: +44 (0) 1245684536
}

\begin{abstract}
This paper introduces an analytical approach to study the textured surfaces in hydrodynamic lubrication regime. For this purpose, a method of integrating the Reynolds equation for slider bearings with surface discontinuities is presented. By introducing appropriate dimensionless parameters, analytical relations for various texture profiles in both indented and projected forms are delivered. These relations express the nature of mathematical dependence between textured bearing performance measures and geometrical/operational parameters. An optimisation procedure is employed to achieve the optimum texturing parameters promoting maximum load capacity, load capacity to lubricant flow rate ratio and minimum friction coefficient for asymmetric partially textured slider bearings.
\end{abstract}

Keywords: Surface texture; Slider bearings; Analytical method; Optimisation

\section{Nomenclature}

$\begin{array}{ll}c_{1}, C_{2} & \text { integration constants } \\ F_{f} & \text { friction force } \\ h & \text { normal distance between two surfaces at any location } \\ h_{D} & \text { normal distance between bearing surfaces at the textured location } \\ h_{d} & \text { maximum height (depth) of the texture measured from texture base } \\ h_{i} & \text { the surface profile of the ith element on the textured surface } \\ h_{m} & \text { minimum normal distance between two parallel flat bearing surfaces } \\ h_{S} & \text { normal distance between two bearing surfaces at any untextured location } \\ H & \text { reference (characteristic) height }\left(=h_{m}\right) \\ L & \text { reference (characteristic) length, bearing length } \\ l & \text { length of the area between two successive textures } \\ l_{d} & \text { length of the texture } \\ l_{0} & \text { length of bearing leading edge }\end{array}$

\footnotetext{
${ }^{1}$ Research Associate, Corresponding Author

${ }^{2}$ Associate Professor

${ }^{3}$ Assistant Professor

${ }^{4}$ Professor of Engineering Design and Simulation
} 
$l_{n} \quad$ length of bearing trailing edge

$m$ number of textures

$p$ pressure

$P_{0} \quad$ pressure at the leading edge

$P_{n} \quad$ pressure at the trailing edge

$Q \quad$ volume flow rate of lubricant

$s \quad$ intermediate integration variable

$S p \quad$ area density of textures

$U$ reference sliding velocity

$U_{1} \quad$ sliding velocity of the lower surface

$u \quad$ velocity profile inside the lubricant film

$W \quad$ load (carrying) capacity

$x, y \quad$ Cartesian (spatial) $x$ - and $y$-coordinates

\section{Greek Symbols}

$\alpha_{0} \quad$ leading edge length to bearing length ratio (leading length ratio)

$\alpha_{\mathrm{n}} \quad$ trailing edge length to bearing length ratio (trailing length ratio)

$\varepsilon \quad$ ratio of textures length to length of area between successive textures

$\eta \quad$ friction coefficient

$\eta_{M} \quad$ modified friction coefficient

$\kappa \quad$ textured area ratio (textured portion)

$\Lambda \quad$ (modified) bearing number

$\Lambda_{F} \quad$ dimensionless friction force

$\Lambda_{p} \quad$ dimensionless pressure

$\Lambda_{Q} \quad$ dimensionless lubricant flow rate

$\Lambda_{W} \quad$ dimensionless load capacity

$\mu \quad$ dynamic viscosity

$\xi \quad$ textures height ratio

$\tau \quad$ shear stress

$\Omega \quad$ dimensionless load capacity to dimensionless lubricant flow rate ratio

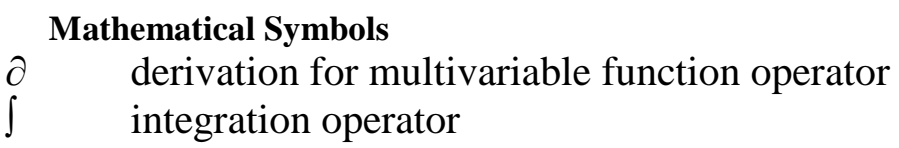

$\Delta \quad$ variations of a parameter

| | absolute value of a parameter

$\rightarrow \quad$ intends to (aims at)

$\infty \quad$ infinity

$\%$ percent

$\sum \quad$ summation operator

$\ln ($ ) natural logarithm

Superscripts

* dimensionless form

\begin{tabular}{ll}
\multicolumn{2}{c}{ Subscripts } \\
$\min$ & minimum \\
$\max$ & maximum \\
opt & optimum
\end{tabular}

Abbreviations 
1D/2D quasi-two dimensional

CFD computational fluid dynamics

FEM finite element method

IT isosceles-triangular

Neg. negative

Pos. positive

RE rectangular

$\mathrm{RT}$ rectangle-triangular

\section{Introduction}

It is now widely accepted that introducing artificially created micro-features onto sliding surfaces can significantly affect friction and wear of the sliding bearings. It is further believed that the textured surfaces provide the best performance amongst all other mechanical surface treatments [1]. The positive effect of artificial surface texturing on enhancing the load capacity, wear resistance and/or friction coefficient of mechanical seals [2], piston-ring/cylinder liner mechanism in internal combustion engines $[3,4,5]$ and roller/piston in hydraulic motors [6] as some of the application examples are shown through numerical and/or experimental studies.

Considering the associated theoretical studies, in a pioneering work by Salama [7], the effect of macro-roughness induced by manufacturing process in the form of surface waviness on the slider bearing performances was studied. As a result, existence of an optimum waviness length to amplitude was reported. By developing a theoretical model to obtain pressure distribution for a single micro-asperity and then, spreading the results for a population of asperities mounted on a radial face seal, Hamilton et al [8] notified existence of an optimum range for asperity height and asperity area fraction providing the maximum loads support. They suggested further research on the effect of interactions amongst asperities. On the other hand, an analytical/experimental study on microasperity lubrication by Anno et al [9] proposed that the poorer correlation between experimental and theoretical results in [8] could be improved by considering the hydrodynamic load support induced by small tilts on the tops of asperities. Later on, Anno et al [10] extended the study to 'negative asperities' and concluded that although all shape of asperities (whether positive or negative) of comparable dimension produce similar load support, to avoid leakage, one must utilise negative asperities.

The study of tribological effects obtained by introducing surface microfeatures was brought to the centre of attention again by Etsion and Burstein [11] who focused on the study of the effect of shallow pores on the operational behaviour of the mechanical face seals. They solved the Reynolds equation numerically using halfSommerfeld boundary condition to predict the performance of mechanical seals comprising hemispherical pores. Later on, the approach was developed to include Swift-Stieber cavitation boundary condition with various pore sizes in terms of having different (maximum) heights [12]. Siripuram [13] compared the hydrodynamic effects of a few deterministic microstructure shapes in mechanical face seal application under the constant load condition through numerical modellings. It was found that the shapes of textures affect leakage or load capacity though having a negligible effect on the friction coefficient. In addition, it was observed that the size and the type of textures (being depressed or projected) do influence the leakage and friction coefficient. However, the comparisons were limited to the results from modelling of only one texture. Razzaque and Faisal [14] have also numerically investigated the 
performance of mechanical face seals with surface micropores in the exponential forms.

A comparison of partially and fully textured surfaces comprising microdimples in parallel thrust bearings has been given by Brizmer et al [15] through employing a numerical approach. After a relatively large number of numerical simulations, they found that the area density of pores, the textured portion, the pores height ratio and the bearing length to width ratio are the most affecting parameters in computing bearing load capacity. Sahlin [16] (also Sahlin et al [17]) investigated the circular and splined shape surface irregularities in relatively high Reynolds numbers using CFD. It was concluded that the load capacity increases with increasing dimple cross sectional area and an optimum for load capacity is achievable when the height ratios lay in the range of 1.5 to 1.75 for all geometries and groove widths. Using a commercial CFD code, Cupillard [18] investigated the effect of introducing a textured surface on the performance of a journal bearing and the mechanism of pressure buildup due to existence of textures in an inclined slider bearing. In addition, the influence of different surface corrugation shapes of triangular, sinusoidal and rectangular and their location on the performance of fixed inclined slider bearings has been studied by Huynh and Loe [19] under non-isothermal flow conditions utilising FEM. Of the three studied corrugation shapes, it was found that rectangle waves produce largest changes in the pressure distribution, normal force and flow rate relative to the smooth walled cases.

Pascovici et al [20,21] have also presented a theoretical investigation of a partially textured slider with square shape dimples wherein the performance of the textured bearing was evaluated by varying different parameters. The analytical approach was based on the flow rate conservation in which the obtained relation for the maximum pressure distribution was based on an assumed pressure distribution profile in partial texturing pattern. In an important work by Wong et al [22], the focus was on adding deterministic textures to the cylinder liner, and study of the effects of such texturing on the hydrodynamic support of the oil ring load as it contributes the majority of ring-pack friction. An averaged Reynolds analysis, using deterministically calculated flow factors, was used to perform a parametric study on both grooved and dimpled textures. Parameters considered for the dimples included diameter, depth, area ratio and arrangement whilst for the grooves width, depth, area ratio, angle and arrangement were considered. They highlighted that it is necessary to understand the effects of different textures, and their relation to slider parameters and running conditions in order to design surfaces for actual tribological applications.

The literature survey indicates that the effect of microfeatures on the generation of hydrodynamic pressure and consequent friction reduction may depend upon textures dimensions, their density and employed pattern on the contacting surfaces [23]. Surface texture shape and orientation may also have a significant effect on the friction and wear. On the other hand, each optimization process requires an accurate correlation of the microgeometry parameters with its induced lubrication influence for the relevant range of operating conditions [22]. Furthermore, study of the literature shows lack of a systematic approach in the study of the textured surfaces and corresponding optimisation procedure. As Etsion [24] also confirms, a trial and error approach is adopted whenever optimization of the texturing dimensions is attempted (e.g. see Brizmer et al [15]). Consequently, a relatively large range of optimum parameters has been attained in different studies. The literature review also reveals that the majority of the theoretical works have been focused on the numerical 
analyses and thus, there is a lack of understanding of the nature of dependence of the interacting parameters with the desired tribological performance measures.

In the present study, the previous model to study the textured surfaces with square form identical irregularities [25] is generalised and further developed to include the explicit analytical relations for various texture types and profiles and present the optimum results based on different performance measures. In this study, the Reynolds equation has been analytically solved for a generic textured contact profile and consequently the analytical relations for bearing performance parameters are obtained. Then using a genetic algorithm search/optimisation method, the optimum geometrical parameters for various texture profiles has been obtained considering different performance parameters. This study provides an analytical approach resulting in the mathematical nature of the relations between geometrical/operational parameters on one hand and the tribological performance parameters for both protrude and depressed textures on the other hand.

\section{General Formulisation of the Problem and Deriving the Associated Analytical Relations}

For a slider bearing with incompressible and isoviscous lubricant, the $1 \mathrm{D} / 2 \mathrm{D}$ form of the steady Reynolds equation can be expressed in the following form ${ }^{5}$ :

$$
\frac{\partial}{\partial x}\left(h^{3}(x) \frac{\partial p(x)}{\partial x}\right)=6 \mu U_{1} \frac{\partial h(x)}{\partial x}
$$

Double integrating of this equation with respect to $x$ will result in the pressure distribution along the bearing surface:

$$
p(x)=6 \mu U_{1} \int_{x_{0}}^{x_{n}} \frac{1}{h(x)^{2}} d x+c_{1} \int_{x_{0}}^{x_{n}} \frac{1}{h(x)^{3}} d x+c_{2}
$$

in which $x_{0}$ and $x_{n}$ are the coordinates of the bearing ends (see Figure 1) and $c_{1}$ and $c_{2}$, the integration constants, can be found from the boundary conditions. The boundary conditions for pressure may be stated as below:

$$
\left\{\begin{array}{l}
\left.p\right|_{x=x_{0}}=0 \\
\left.p\right|_{x=x_{n}}=P_{n}-P_{0}
\end{array}\right.
$$

where the pressure at the starting point of the bearing is set to zero and instead a gauge pressure at the ending point is used. Using these boundary conditions, the integration constants would be determined as follows:

$$
\left\{\begin{array}{l}
c_{1}=\frac{\left(P_{n}-P_{0}\right)-6 \mu U_{1} \int_{x_{0}}^{x_{n}} \frac{1}{h(x)^{2}} d x}{\int_{x_{0}}^{x_{n}} \frac{1}{h(x)^{3}} d x} \\
c_{2}=0
\end{array}\right.
$$

In general, the surface profile for a bearing including surface irregularities can be defined with a piecewise form function as below (see Figure 1):

\footnotetext{
${ }^{5}$ It is noted that in the present study the bearing and the textures are both assumed infinite in width.
} 


$$
h(x)=\left\{\begin{array}{lc}
h_{1}(x) ; & x_{0} \leq x<x_{1} \\
h_{2}(x) ; & x_{1} \leq x<x_{2} \\
\vdots & \vdots \\
h_{n}(x) ; & x_{n-1} \leq x \leq x_{n}
\end{array}\right.
$$

\section{Figure 1}

Using this sample surface profile, the pressure distribution for any distinctive section of the bearing can be expressed in the following form:

$$
\begin{aligned}
\left.p(x)\right|_{x \in\left[x_{i-1}, x_{i}\right]}=6 & \mu U_{1}\left(\int_{x_{0}}^{x_{1}} \frac{1}{h_{1}(x)^{2}} d x+\int_{x_{1}}^{x_{2}} \frac{1}{h_{2}(x)^{2}} d x+\cdots \int_{x_{i-1}}^{x_{i}} \frac{1}{h_{i}(x)^{2}} d x\right) \\
& +c_{1}\left(\int_{x_{0}}^{x_{1}} \frac{1}{h_{1}(x)^{3}} d x+\int_{x_{1}}^{x_{2}} \frac{1}{h_{2}(x)^{3}} d x+\cdots \int_{x_{i-1}}^{x_{i}} \frac{1}{h_{i}(x)^{3}} d x\right)+c_{2}
\end{aligned}
$$

In addition, the integration constant $c_{1}$ can be determined as follows:

$$
c_{1}=\frac{\left(P_{n}-P_{0}\right)-6 \mu U_{1}\left(\int_{x_{0}}^{x_{1}} \frac{1}{h_{1}(x)^{2}} d x+\int_{x_{1}}^{x_{2}} \frac{1}{h_{2}(x)^{2}} d x+\cdots \int_{x_{n-1}}^{x_{n}} \frac{1}{h_{n}(x)^{2}} d x\right)}{\left(\int_{x_{0}}^{x_{1}} \frac{1}{h_{1}(x)^{3}} d x+\int_{x_{1}}^{x_{2}} \frac{1}{h_{2}(x)^{3}} d x+\cdots \int_{x_{n-1}}^{x_{n}} \frac{1}{h_{n}(x)^{3}} d x\right)}
$$

Having the pressure distribution, the load capacity of the bearing is calculated using the pressure distribution as below:

$$
W=\int_{x_{0}}^{x_{n}} p(x) d x=\left.\int_{x_{0}}^{x_{1}} p(x)\right|_{x \in\left[x_{0}, x_{1}\right]} d x+\left.\int_{x_{1}}^{x_{2}} p(x)\right|_{x \in\left[x_{1}, x_{2}\right]} d x+\left.\cdots \int_{x_{n-1}}^{x_{n}} p(x)\right|_{x \in\left[x_{n-1}, x_{n}\right]} d x
$$

In order to introduce the associated relation for the friction force, the velocity profile inside the lubricant film that is [26]:

$$
u(x, y)=\frac{1}{2 \mu}\left(\frac{\partial p(x)}{\partial x}\right)\left(y^{2}-h(x) y\right)+U_{1}\left(\frac{h(x)-y}{h(x)}\right)
$$

is employed to determine the shear stress exerted on the lower surface which is generally defined as below:

$$
\tau(x)=\left.\mu \frac{\partial u(x, y)}{\partial y}\right|_{y=0}
$$

Replacing the velocity profile from equation (9) and the pressure gradient, which is calculated from equation (6), into equation (10), would result in the shear stress experienced by the lower surface in any distinctive area, as below:

$$
\left.\tau(x)\right|_{x \in\left[x_{i-1}, x_{i}\right]}=-\left(4 \mu U_{1} \frac{1}{h_{i}(x)}+\frac{c_{1}}{2} \frac{1}{h_{i}(x)^{2}}\right)
$$

Using the obtained form for the shear stress from equation (11) and integrating it for the entire bearing length, the friction force will be: 


$$
F_{f}=\int_{x_{0}}^{x_{n}} \tau(x) d x=\left.\int_{x_{0}}^{x_{1}} \tau(x)\right|_{x \in\left[x_{0}, x_{1}\right]} d x+\left.\int_{x_{1}}^{x_{2}} \tau(x)\right|_{x \in\left[x_{1}, x_{2}\right]} d x+\left.\cdots \int_{x_{n-1}}^{x_{n}} \tau(x)\right|_{x \in\left[x_{n-1}, x_{n}\right]} d x
$$

Now, the friction coefficient can be defined as:

$$
\eta=\frac{F_{f}}{W}
$$

in which $F_{f}$ and $W$ are replaced from equations (12) and (8), respectively.

Finally, the volume flow rate of lubricant in each cross-section can be calculated by integrating the velocity profile from equation (9) resulting in the following relation:

$$
Q=-\frac{1}{12 \mu}\left(\frac{\partial p}{\partial x}\right)_{i} h_{i}(x)^{3}+\frac{1}{2} U_{1} h_{i}(x)
$$

Considering the pressure distribution in equation (6) and the specified notions in Figure 2, equation (8) for the load capacity can be written as follows:

$$
\begin{aligned}
W & =\left(\int_{0}^{l_{0}} \int_{0}^{x} \frac{1}{h_{S}^{2}} d s d x+\int_{0}^{l_{n}} \int_{0}^{x} \frac{1}{h_{S}^{2}} d s d x+l_{n} \int_{0}^{l_{0}} \frac{1}{h_{S}^{2}} d x\right)+m\left(l_{d} \int_{0}^{l_{0}} \frac{1}{h_{S}^{2}} d x+\int_{0}^{l_{d}} \int_{0}^{x} \frac{1}{h_{D}{ }^{2}} d s d x+l_{n} \int_{0}^{l_{d}} \frac{1}{h_{D}{ }^{2}} d x\right) \\
& +(m-1)\left(l \int_{0}^{l_{0}} \frac{1}{h_{S}^{2}} d x+\int_{0}^{l} \int_{0}^{x} \frac{1}{h_{S}^{2}} d s d x+l_{n} \int_{0}^{l} \frac{1}{h_{S}^{2}} d x\right)+\frac{(m-1)(m-2)}{2}\left(l \int_{0}^{l} \frac{1}{h_{S}^{2}} d x\right) \\
& +\frac{m(m-1)}{2}\left(l \int_{0}^{l_{d}} \frac{1}{h_{D}^{2}} d x+l_{d} \int_{0}^{l_{d}} \frac{1}{h_{D}^{2}} d x+l_{d} \int_{0}^{l} \frac{1}{h_{S}^{2}} d x\right)
\end{aligned}
$$

By following an analogous approach, the friction force for identical textures can be written in the form presented below:

$$
F_{f}=-4 \mu U_{1}\left(\int_{0}^{l_{0}} \frac{d x}{h_{S}}+m \int_{0}^{l_{d}} \frac{d x}{h_{D}}+(m-1) \int_{0}^{l} \frac{d x}{h_{S}}+\int_{0}^{l_{n}} \frac{d x}{h_{S}}\right)-\frac{c_{1}}{2}\left(\int_{0}^{l_{0}} \frac{d x}{h_{S}{ }^{2}}+m \int_{0}^{l_{d}} \frac{d x}{h_{D}{ }^{2}}+(m-1) \int_{0}^{l} \frac{d x}{h_{S}{ }^{2}}+\int_{0}^{l_{n}} \frac{d x}{h_{S}{ }^{2}}\right)
$$

Now, the general relations delivered above in integral form as well as the given relation for lubricant flow rate in equation (14) can be theoretically employed for analysis of any given texture and/or bearing surface profile.

\section{Figure 2}

Considering Figure 2, the leading edge length to bearing length ratio (leading length ratio) and the trailing edge length to bearing length ratio (trailing length ratio) can be defined as follows, respectively:

$$
\alpha_{0}=\frac{l_{0}}{L}, \quad \alpha_{n}=\frac{l_{n}}{L}
$$

Therefore, the textured length or area ratio (textured portion) of the bearing will be:

$$
\kappa=1-\left(\alpha_{0}+\alpha_{n}\right)
$$

In addition, the ratio of textures length to distance between textures or correspondingly the area density of the textures can be defined as below, respectively:

$$
\varepsilon=\frac{l_{d}}{l}, \quad S p=\frac{l_{d}}{l+l_{d}}
$$

The ratio of textures length to distance between textures (textures length to distance or simply textures length ratio) and the area density of the textures can be related to each other as follows: 


$$
S p=\frac{\varepsilon}{1+\varepsilon}
$$

Eventually, the ratio of maximum to minimum distances between bearing surfaces (or the textures height ratio) can also be defined as:

$$
\xi=\frac{h_{D}}{h_{S}}=\frac{h_{m}+h_{d}}{h_{m}}=1+\frac{h_{d}}{h_{m}}
$$

Now, the above-mentioned dimensionless parameters can be utilised to derive the analytical relations for the specified form of the texture profiles in both negative and positive types ${ }^{6}$.

A surface comprising negative rectangular shape textures, which is shown in Figure 2, can be defined as follows:

$$
h(x)= \begin{cases}h_{m} ; & x_{0} \leq x \leq x_{1} \\ h_{m}+h_{d} ; & x_{1}<x \leq x_{2} \\ \vdots & \vdots \\ h_{m} ; & x_{n-1}<x \leq x_{n}\end{cases}
$$

Considering Figure 2 and the given surface profile for negative rectangular textures in equation (22) as well as employing the defined dimensionless geometrical parameters in equations (17) to (21), the following analytical dimensionless relations can be achieved for the load capacity, friction force and lubricant flow rate, respectively,

$$
\begin{aligned}
\Lambda_{W}= & \frac{3 m \varepsilon \kappa\left(1-\kappa-2 \alpha_{n}\right)(\xi-1)}{(m+\varepsilon-1) \xi^{3}-m \varepsilon \kappa\left(\xi^{3}-1\right)}+\left(\frac{\Lambda}{2}\right) \frac{(m+\varepsilon-1) \xi^{3}-m \varepsilon \kappa\left(\kappa+2 \alpha_{n}\right)\left(\xi^{3}-1\right)}{(m+\varepsilon-1) \xi^{3}-m \varepsilon \kappa\left(\xi^{3}-1\right)} \\
\Lambda_{F} & =\frac{m^{2} \varepsilon^{2} \kappa^{2}(\xi-1)^{4}+\xi(m+\varepsilon-1)\left[(m+\varepsilon-1) \xi^{3}-2 m \varepsilon \kappa(\xi-1)\left(\xi^{2}-\xi+2\right)\right]}{\xi(m+\varepsilon-1)\left[(m+\varepsilon-1) \xi^{3}-m \varepsilon \kappa\left(\xi^{3}-1\right)\right]} \\
& -\left(\frac{\Lambda}{2}\right) \frac{\xi\left[(m+\varepsilon-1) \xi^{2}-m \varepsilon \kappa\left(\xi^{2}-1\right)\right]}{(m+\varepsilon-1) \xi^{3}-m \varepsilon \kappa\left(\xi^{3}-1\right)}
\end{aligned}
$$

and

$$
\Lambda_{Q}=-\frac{\xi\left[(m+\varepsilon-1) \xi^{2}-m \varepsilon \kappa\left(\xi^{2}-1\right)\right]}{2\left[(m+\varepsilon-1) \xi^{3}-m \varepsilon \kappa\left(\xi^{3}-1\right)\right]}-\left(\frac{\Lambda}{12}\right) \frac{(m+\varepsilon-1) \xi^{3}}{\left[(m+\varepsilon-1) \xi^{3}-m \varepsilon \kappa\left(\xi^{3}-1\right)\right]}
$$

where $\Lambda_{W}, \Lambda_{F}$ and $\Lambda_{Q}$ are:

$$
\Lambda_{W}=\frac{W H^{2}}{\mu U L^{2}}, \quad \Lambda_{F}=\frac{F H}{\mu U L}, \quad \Lambda_{Q}=\frac{Q}{U H}
$$

and the bearing number, $\Lambda$, is defined as below:

\footnotetext{
${ }^{6}$ In the present study, the term 'negative' will be used if the surface irregularities are in the form of depressions, which are made by cutting into the surface, and correspondingly the term 'positive' will be used if the surface irregularities are in the form of projected objectives or protrudes. In addition, the term 'type' will merely be used in order to clarify whether the surface irregularities are 'negative' or 'positive' whilst the term 'profile' will solely be used when the shape of textures are considered. In addition, the term 'height' will be considered equivalent to the 'depth' for the 'negative type textures'.
} 


$$
\Lambda=\frac{\left(P_{n}-P_{0}\right) H^{2}}{\mu U L}
$$

In addition, the dimensionless pressure inside the lubricant film can be defined as follows:

$$
\Lambda_{p}=\frac{\left(p-P_{0}\right) H^{2}}{\mu U L}
$$

Note that in the corresponding relations for negative type textures, without loss of the generality of problem, it was assumed that $U=-U_{1}$ in order to have a dominant positive pressure distribution for the given configurations in this study.

Subsequently, the 'modified' friction coefficient, $\eta_{M}$, can be obtained considering equations (13), (23) and (24):

$$
\eta_{M}=\eta\left(\frac{L}{H}\right)
$$

In addition, the ratio of dimensionless load capacity to dimensionless lubricant flow rate, $\Omega$; can also be defined in order to bring the lubricant consumption into account when optimisation is aimed. This parameter is defined as follows:

$$
\Omega=\frac{\Lambda_{W}}{\Lambda_{Q}}
$$

As can be seen from equations (23) to (25), the variables are textured length ratio, $\kappa$, textures height ratio, $\xi$, number of textures, $m$, textures length to distance ratio, $\varepsilon$, trailing length ratio, $\alpha_{n}$ and finally bearing number, $\Lambda$. It is noted that the relations above could also be derived using Sp instead of $\varepsilon$ and $\alpha_{0}$ instead of $\kappa$; however, during derivation of the equations it was observed that using the latter parameters the equations could be delivered in more complicated form.

Figures $3 a$ and $b$ show schematic of a slider bearing comprising identical isosceles-triangular and rectangle-triangular shape textures, respectively. The surface profiles can be defined as follows:

$$
\begin{cases}h(x)=h_{m} ; & \text { for untextured area } \\ h(x)=h_{m}+\left(\frac{h_{d}}{l_{d} / 2}\right) x ; & \text { for the first - half of triangle } \\ h(x)=h_{m}+h_{d}-\left(\frac{h_{d}}{l_{d} / 2}\right) x ; & \text { for the second - half of triangle }\end{cases}
$$

for isosceles-triangular and,

$$
\begin{cases}h(x)=h_{m} ; & \text { for untextured area } \\ h(x)=h_{m}+\left(\frac{h_{d}}{l_{d}}\right) x ; & \text { for textured area }\end{cases}
$$

for rectangle-triangular shape textures.

\section{Figure 3}

The corresponding equations representing the dimensionless load capacity for isosceles-triangular and rectangle-triangular negative textures are derived as follows, respectively: 


$$
\begin{aligned}
\Lambda_{W} & =\frac{3 m \varepsilon \kappa\left(1-\kappa-2 \alpha_{n}\right)(\xi-1)}{2(m+\varepsilon-1) \xi^{2}-m \varepsilon \kappa(2 \xi+1)(\xi-1)} \\
& +\left(\frac{\Lambda}{2}\right) \frac{2(m+\varepsilon-1) \xi^{2}-m \varepsilon \kappa\left(\kappa+2 \alpha_{n}\right)(2 \xi+1)(\xi-1)}{2(m+\varepsilon-1) \xi^{2}-m \varepsilon \kappa(2 \xi+1)(\xi-1)}
\end{aligned}
$$

and

$$
\begin{aligned}
\Lambda_{W} & =\frac{6 m \varepsilon^{2} \kappa^{2}}{(m+\varepsilon-1)^{2}(\xi-1)^{2}} \ln (\xi) \\
& +\frac{3 m \varepsilon \kappa\left\{\begin{array}{l}
m \varepsilon^{2} \kappa^{2}(\xi+5)(\xi-1)-\kappa(m+\varepsilon-1)\left[(m-1)(\xi-1)^{2}+2 \varepsilon \xi(\xi+1)\right] \\
-(m+\varepsilon-1)^{2}(\xi-1)^{2}\left(2 \alpha_{n}-1\right)
\end{array}\right\}}{(m+\varepsilon-1)^{2}(\xi-1)\left[2(m+\varepsilon-1) \xi^{2}-m \varepsilon \kappa(2 \xi+1)(\xi-1)\right]} \\
& +\left(\frac{\Lambda}{2}\right) \frac{(m+\varepsilon-1)\left\{\begin{array}{l}
2(m+\varepsilon-1) \xi^{2} \\
-m \varepsilon \kappa\left[(2 \xi-1) \xi\left(\kappa+2 \alpha_{n}\right)-2 \alpha_{n}\right]
\end{array}\right\}+m \varepsilon \kappa^{2}(m+\xi \varepsilon-1)}{(m+\varepsilon-1)\left[2(m+\varepsilon-1) \xi^{2}-m \varepsilon \kappa(2 \xi+1)(\xi-1)\right]}
\end{aligned}
$$

Despite of the difference in the obtained dimensionless load capacities, the corresponding relations for dimensionless friction force and lubricant flow rate are similar for both of the triangular form textures:

and

$$
\begin{aligned}
\Lambda_{F} & =\frac{4 m \varepsilon \kappa}{(\xi-1)(m+\varepsilon-1)} \ln (\xi)-\Lambda \frac{\xi[(m+\varepsilon-1) \xi-m \varepsilon \kappa(\xi-1)]}{2(m+\varepsilon-1) \xi^{2}-m \varepsilon \kappa\left(2 \xi^{2}-\xi-1\right)} \\
& +\frac{2\left\{(m+\varepsilon-1)\left[(m+\varepsilon-1) \xi^{2}-2 m \varepsilon \kappa\left(\xi^{2}+2 \xi-1\right)\right]+m^{2} \varepsilon^{2} \kappa^{2}(\xi+5)(\xi-1)\right\}}{(m+\varepsilon-1)\left[2(m+\varepsilon-1) \xi^{2}-m \varepsilon \kappa\left(2 \xi^{2}-\xi-1\right)\right]}
\end{aligned}
$$

$$
\Lambda_{Q}=-\frac{\xi[(m+\varepsilon-1) \xi-m \varepsilon \kappa(\xi-1)]}{\left[2(m+\varepsilon-1) \xi^{2}-m \varepsilon \kappa(2 \xi+1)(\xi-1)\right]}-\left(\frac{\Lambda}{6}\right) \frac{(m+\varepsilon-1) \xi^{2}}{\left[2(m+\varepsilon-1) \xi^{2}-m \varepsilon \kappa(2 \xi+1)(\xi-1)\right]}
$$

Therefore, for triangular shape textures, the form of the triangular (having right angle or being isosceles) seems to be an insignificant parameter in calculating the friction force and lubricant flow rate. Additionally, for all of the studied texture profiles so far it can be observed that, unlike for the load capacity, the trailing length ratio, $\alpha_{n}$, does not appear directly in the obtained relations for the friction force and lubricant flow rate and its existence or absence can only be implicitly influential by affecting $\kappa$.

In a rather similar fashion, it is possible to extend the relations for positive textures. Figures 4a, b and c illustrate the rectangular, isosceles-triangular and rectangle-triangular textures in positive (protrude) form, respectively. The similar dimensionless parameters used for negative textures are also applicable here without any alterations except that $U=U_{1}$ in order to have dominant positive pressure distribution for the given configurations in this study.

\section{Figure 4}

The dimensionless load capacities for positive rectangular, isosceles-triangular and rectangle-triangular textures are as follows, respectively:

$$
\Lambda_{W}=\frac{3 m \varepsilon \kappa\left(1-\kappa-2 \alpha_{n}\right)(\xi-1)}{(m+\varepsilon-1)+m \varepsilon \kappa\left(\xi^{3}-1\right)}+\left(\frac{\Lambda}{2}\right) \frac{(m+\varepsilon-1)+m \varepsilon \kappa\left(\kappa+2 \alpha_{n}\right)\left(\xi^{3}-1\right)}{(m+\varepsilon-1)+m \varepsilon \kappa\left(\xi^{3}-1\right)}
$$




$$
\Lambda_{W}=\frac{3 m \varepsilon \kappa\left(1-\kappa-2 \alpha_{n}\right)(\xi-1)}{\xi[2(m+\varepsilon-1)+m \varepsilon \kappa(\xi+2)(\xi-1)]}+\left(\frac{\Lambda}{2}\right) \frac{2(m+\varepsilon-1)+m \varepsilon \kappa\left(\kappa+2 \alpha_{n}\right)(\xi+2)(\xi-1)}{2(m+\varepsilon-1)+m \varepsilon \kappa(\xi+2)(\xi-1)}
$$

and

$$
\begin{aligned}
\Lambda_{W} & =\frac{6 m \varepsilon^{2} \kappa^{2}}{(m+\varepsilon-1)^{2}(\xi-1)^{2}} \ln (\xi) \\
& -\frac{3 m \varepsilon \kappa\left\{\begin{array}{l}
m \varepsilon^{2} \kappa^{2}(5 \xi+1)(\xi-1)+2 \varepsilon \kappa(m+\varepsilon-1)(\xi+1) \\
+(m+\varepsilon-1)(\xi-1)^{2}\left[(m-1) \kappa+(m+\varepsilon-1)\left(2 \alpha_{n}-1\right)\right]
\end{array}\right\}}{\xi(m+\varepsilon-1)^{2}(\xi-1)[2(m+\varepsilon-1)+m \varepsilon \kappa(\xi+2)(\xi-1)]} \\
& +\left(\frac{\Lambda}{2}\right) \frac{m \varepsilon \kappa(\xi+2)(\xi-1)\left[\begin{array}{l}
(m-1)\left(\kappa+2 \alpha_{n}\right) \\
+2 \varepsilon \alpha_{n}
\end{array}\right]+2 m \varepsilon^{2} \kappa^{2}(\xi-1)+2(m+\varepsilon-1)^{2}}{(m+\varepsilon-1)[2(m+\varepsilon-1)+m \varepsilon \kappa(\xi+2)(\xi-1)]}
\end{aligned}
$$
below:

In addition, the friction force for positive rectangular textures can be recast as

$$
\begin{aligned}
\Lambda_{F} & =-\frac{m^{2} \varepsilon^{2} \kappa^{2}(\xi-1)^{4}+2(m+\varepsilon-1)\left[(m+\varepsilon-1)+2 m \varepsilon \kappa(\xi-1)\left(2 \xi^{2}-\xi+1\right)\right]}{\xi(m+\varepsilon-1)\left[(m+\varepsilon-1)+m \varepsilon \kappa\left(\xi^{3}-1\right)\right]} \\
& -\left(\frac{\Lambda}{2}\right) \frac{\xi\left[(m+\varepsilon-1)+m \varepsilon \kappa\left(\xi^{2}-1\right)\right]}{(m+\varepsilon-1)+m \varepsilon \kappa\left(\xi^{3}-1\right)}
\end{aligned}
$$

As was already seen, both of the negative triangular form textures had identical relations for friction force. It is so here as well and the friction force for both of positive triangular form textures is:

$$
\begin{aligned}
\Lambda_{F} & =-\frac{4 m \varepsilon \kappa}{\xi(\xi-1)(m+\varepsilon-1)} \ln (\xi)-\Lambda \frac{\xi[(m+\varepsilon-1)+m \varepsilon \kappa(\xi-1)]}{2(m+\varepsilon-1)+m \varepsilon \kappa(\xi+2)(\xi-1)} \\
& -\frac{2\left\{(m+\varepsilon-1)\left[(m+\varepsilon-1)+2 m \varepsilon \kappa\left(\xi^{2}-2 \xi-1\right)\right]-m^{2} \varepsilon^{2} \kappa^{2}(5 \xi+1)(\xi-1)\right\}}{\xi(m+\varepsilon-1)[2(m+\varepsilon-1)+m \varepsilon \kappa(\xi+2)(\xi-1)]}
\end{aligned}
$$

Moreover, the dimensionless lubricant flow rate for positive rectangular textures can be expressed as follows:

$$
\Lambda_{Q}=\frac{\xi\left[(m+\varepsilon-1)+m \varepsilon \kappa\left(\xi^{2}-1\right)\right]}{2\left[(m+\varepsilon-1)+m \varepsilon \kappa\left(\xi^{3}-1\right)\right]}-\left(\frac{\Lambda}{12}\right) \frac{(m+\varepsilon-1) \xi^{3}}{\left[(m+\varepsilon-1)+m \varepsilon \kappa\left(\xi^{3}-1\right)\right]}
$$

Finally, the lubricant flow rate for positive rectangle-triangular and isoscelestriangular shape textures will be:

$$
\Lambda_{Q}=\frac{\xi[(m+\varepsilon-1)+m \varepsilon \kappa(\xi-1)]}{[2(m+\varepsilon-1)+m \varepsilon \kappa(\xi+2)(\xi-1)]}-\left(\frac{\Lambda}{6}\right) \frac{(m+\varepsilon-1) \xi^{3}}{[2(m+\varepsilon-1)+m \varepsilon \kappa(\xi+2)(\xi-1)]}
$$

Now, the general form of analytical relations for load capacity, friction force (and hence, friction coefficient), and lubricant flow rate (and hence, the ratio of dimensionless load capacity to dimensionless lubricant flow rate) are delivered for all three different texture profiles in both negative and positive types. These relations include variations of different geometrical parameters as well as variations in the pressure at boundaries.

\section{The Optimisation Results and Discussion}

As the derived equations indicate, due to the non-linearity of some of the equations because of existence of transcendental function, a non-linear optimisation approach must be employed. Thus, a robust genetic algorithm search/optimisation 
method was developed to tackle the problem, which also enables to find the optimum of multiple variables simultaneously.

Amongst the parameters considered in this study, $\Lambda$ is dependent to the pressure difference in the boundaries and hence is not a geometrical parameter. Thus, it can be investigated independently. Therefore, first the optimisation results will be revealed for $\Lambda=0$ as a common choice indicating no pressure difference between the bearing ends. Then, the effect of variations of $\Lambda$ on the optimum parameters will be investigated anon.

In addition, since the trailing edge has always a negative effect on the textured bearing performance by producing negative pressures [25], by reducing the corresponding trailing edges for both negative and positive textures, one can reduce this negative effect. Figures $5 a$ and $b$ demonstrate the variations of dimensionless pressure distribution inside the lubricant film for two series of tests. To obtain the results, four negative rectangular textures were employed on a slider bearing surface with arbitrary $\xi=2.0$ and $S p=0.4$. In the first series of tests (see Figure 5a), by keeping $\kappa$ constant, $\alpha_{n}$ was reduced uniformly and since the bearing length was constant too, therefore $\alpha_{0}$ was increased correspondingly. In the second series of tests (see Figure 5b), $\alpha_{0}$ was kept constant and as $\alpha_{n}$ was reducing, $S p$ was increased correspondingly to maintain the constant bearing length. As can be seen through the results in Figure 5, in both series, by reducing the length of the trailing edges the size of the region with negative pressure, which is an indicator of occurrence of cavitation, reduces.

Therefore, in the rest of the study, asymmetrical partially textured slider bearings with $\alpha_{n}=0$ will be considered which in turn would result in the least possibility for occurrence of cavitation at the rear end especially at the optimum configuration. This indeed, provides results with practical importance.

\section{Figure 5}

It is noted that the optimisation will be conducted considering three objective functions of $\Lambda_{W}, \Omega$ and $\eta_{M}$, where the first two will be maximised whilst the latter will be minimised. Preliminary examinations of the obtained equations for the load capacity, friction coefficient and load capacity to lubricant flow ratio for all texture shapes considered in this study, based on the number of textures, $m$, and texture length to distance ratio, $\varepsilon$, shows no specific real extremum points. In fact, by increasing the number of textures and/or reducing the area density of textures, $\Lambda_{W}$ and $\Omega$ reduce whilst $\mu_{M}$ increases uniformly regardless of the variations of the other independent variables in the given range. This result is in line with the previous observations that in the case of $m=1$ or $S p=1$, the resultant bearing would be the Rayleigh step which is known as the bearing with the best tribological performance amongst all other bearing surface profiles (see [27]). Therefore, to find a typical set of optimum value(s) for the rest of independent variables a constant textures length to distance ratio will be assumed. However, variations of the obtained optimum values with the area density of textures will be investigated later on.

\subsection{Optimisation results for $\Lambda=0$}

Considering $\Lambda=0$ and $S p=0.5$ as a typical value for area density of textures, the obtained optimum height and textured area ratios versus the number of textures based on maximisation of load capacity are demonstrated in Figure 6 for all three studied texture profiles in both negative and positive types. As can be seen from Figure 6a, although the optimum height ratio, $\xi_{\text {opt }}$, reduces drastically for negative textures at the 
lower $m$ values, it varies rather slightly by increasing the number of textures. An interesting point about the optimum height ratio for negative rectangle-triangular textures is that $\xi_{\text {opt }}$ increases (very slightly) with the number of textures of greater than two whilst it is not so for the other negative textures. For positive textures, the optimum height ratio grows with the number of textures. Apart from the negative rectangular textures, the optimum height ratios for the rest of cases become closer to each other at higher texture numbers. Figure $6 \mathrm{a}$ also indicates that for the lesser number of textures, $\xi_{\text {opt }}$ may vary considerably by changing textures profiles and/or types. In addition, the negative isosceles-triangular textures have the highest optimum values when there is a few number of textures whilst by raising of $m$, both of the positive triangular form textures demand higher $\xi_{\text {opt }}$ so that both have the highest optimum values. On the other hand, the negative rectangular textures have the lowest height ratios at all number of textures, which are also considerably less than the corresponding optimum values for the other textures. Another important point, which can be observed from Figure 6a, is that although the difference between the two negative triangular form textures decreases with $m$, their positive equivalents have almost similar optimum values at all number of textures except at $m=1$.

Comparing the optimum textured area ratios shown in Figure $6 \mathrm{~b}$ indicates higher optimum values for negative textures even though $\kappa_{\text {opt }}$ for this type of textures reduces slightly by increasing the number of textures. The difference in the optimum $\kappa$ for different negative texture profiles also diminishes by increasing $m$. In fact, by growing the number of textures, $\kappa_{\text {opt }}$ for negative textures remains independent of the textures profiles and is about 0.55 . For positive textures, $\kappa_{\text {opt }}$ is relatively lower and the influence of the shape of textures is considerable even at higher number of textures. For lower number of textures, although both of the positive triangular form textures show considerable difference in the trend of variations, their optimum values converge to each other by increasing $m$; taking an optimum value of about 0.40 ultimately. The optimum textured area ratio for the positive rectangular textures is the lowest of all at all number of textures and reaches to about 0.3 for higher number of textures. It is also noted that for $m=1$, the optimum textured area ratio is in fact, an indicator of the optimum texture length ratio. In this regard, the positive rectangletriangular texture has the highest optimum value of about 0.87 .

The obtained maximum load capacities are demonstrated in Figure 6c in the dimensionless form. As the results in this figure suggest, the highest $\left(\Lambda_{W}\right)_{\max }$ belongs to the positive rectangular textures. In general, the obtained maximum load capacities reduce as the number of textures rises up. This decline is more considerable at lower number of textures. For $m=1$ and 2 the positive isosceles-triangular and for the rest, the negative isosceles- as well as rectangle-triangular textures have the lowest maximum dimensionless load capacities whilst the negative rectangular textures have the second highest maximum load capacities. Overall, Figure 6c reveals that in terms of the load capacity, the positive type of textures perform better than their corresponding negative ones.

\section{Figure 6}

In addition to the load capacity, minimisation of the friction coefficient can also be of interest. The results for optimum height ratio and textured area ratio providing the minimum friction coefficient in each case are presented in Figures 7a and b, respectively. As the results in Figure 7a illustrate, the general trends in the variations of $\xi_{\mathrm{opt}}$ with $m$ for different considered texture types and profiles remain 
almost similar to the previous results shown in Figure 6a. However, there are differences in the order in which some of the results appear. For instance, by increasing $m$, the difference in $\xi_{\text {opt }}$ between corresponding positive and negative triangular textures rises noticeably. In fact, the positive type of both of the triangular form textures here, demand much higher $\xi_{\mathrm{opt}}$ by increasing the number of textures.

As Figure $7 \mathrm{~b}$ demonstrates, the obtained optimum values for $\kappa$ have also very similar trend comparing their corresponding in Figure $6 \mathrm{~b}$ above obtained based on maximisation of the load capacity. Nevertheless, there are some differences in the obtained values. For instance, although the positive rectangular textures here also have the lowest $\kappa_{\text {opt }}$ of about 0.21 at higher number of textures, it is somehow less than 0.3 , which was obtained considering maximisation of the load capacity.

The resulting minimum modified friction coefficients for the studied texture profiles and types are given in Figure 7c. As this figure indicates, in all cases, $\left(\eta_{M}\right)_{\min }$ increases with the number of textures although for $m>1$, the positive textures have much lower minimum modified friction coefficient than the negative textures. The increment in the $\left(\eta_{M}\right)_{\min }$ with the number of textures is more considerable for negative textures. It is important to note that comparing the results in this figure with the results shown in Figure 6c for the maximum load capacity points towards the fact that the difference in the results here is rather significantly influenced by the type and shape of textures. As Figure 7c also reveals, the positive rectangular textures have the lowest and the negative types of both of triangular textures have the highest minimum modified friction coefficients.

\section{Figure 7}

The ratio of dimensionless load capacity to dimensionless lubricant flow rate was already defined to facilitate considering the lubricant consumption in producing the load capacity. Figures $8 \mathrm{a}$ and $\mathrm{b}$ demonstrate the optimum height and textured area ratios obtained by maximisation of $\Omega$, respectively. As Figure 8a shows, for $m>1$, the negative rectangular textures have the lowest optimum height ratios despite the fact that the rest of negative type textures have higher optimum values than the positive ones. Additionally, the optimum height ratios for the triangular profile textures of the same type converge to each other as the number of textures increase whilst the difference in the obtained optimum values for positive and negative rectangular textures are considerably high. In fact, considering $\Omega$, the influence of shape and type of textures on the optimum height ratio becomes distinguishable.

Comparing the variations of $\kappa_{\mathrm{opt}}$ shown in Figure 8b with the corresponding variations of $\kappa_{\text {opt }}$ in Figures $6 \mathrm{~b}$ and $7 \mathrm{~b}$, shows that the trend in the variations of $\kappa_{\mathrm{opt}}$ is not a vary strong function of $m$ or textures type and/or shape although, the obtained $\kappa_{\text {opt }}$ for each case can change even noticeably at very low number of textures. The results also indicate that for the negative textures, the shape of textures has a negligible effect on $\kappa_{\text {opt }}$ whilst for the positive textures the effect is still significant. The obtained optimum textured area ratio for positive rectangle-triangular textures based on maximisation of $\Omega$ specifies that at $m=1$ the optimum textured surface becomes in fact an inclined sliding pad.

Figure 8c demonstrates the variations of $\Omega_{\max }$ with the number of textures where the positive rectangular textures have the highest maximum values whilst in contrast, the rest of the positive textures possess the lowest maximum values for all studied number of textures. Meanwhile, the results obtained for negative textures take place in between, with higher values for rectangular textures. The value of $\Omega_{\max }$ also 
reduces with $m$ and the difference between isosceles- and rectangle-triangular textures of the same type shrinks as the number of textures grows.

\section{Figure 8}

\subsection{Justifying the model and assumptions}

At the beginning, it was assumed that by minimising the length of the trailing edge of a textured bearing one could avoid the occurrence of cavitation in the asymmetrically partially textured bearing or at least reduce it to a very trivial amount, which would virtually have no effect on the results obtained in the current form of analyses. This idea could be strengthen by assuming that in the optimum configuration the obtained geometry should naturally become in a state that automatically eliminates or at least minimises the chance of occurrence of cavitation so that all parts of the bearing contribute towards producing the maximum performance.

As now the optimum geometrical parameters in each studied texture number, type and profile is known, hence it seems to be adequate to investigate this assumption by examining the corresponding pressure distribution inside the bearing. Here, as an example, the pressure distribution inside a bearing comprising five textures in all studied types and profiles will be examined. The relevant results for the dimensionless pressure distribution are shown in Figure 9a. The results clearly support the earlier assumption. However, further examinations show that by reducing the number of textures, the area with negative pressure at the rear end of bearing may grow slightly in the case of isosceles-triangular textures. The most noticeable negative pressure is produced in the case of a bearing with only one negative or positive isosceles-triangular texture. Nonetheless, by increasing the number of textures to two this area with negative pressure becomes much smaller. Figure 9b compares the dimensionless pressure distributions for a bearing with one and two negative and positive isosceles-triangular textures.

\section{Figure 9}

\subsection{The implication of optimisation based on different objective functions}

As the obtained results so far can also be evidence for, quite naturally, by altering the type of objective function the corresponding optimum geometrical parameters can change. To evaluate this quantitatively, considering the optimum results for maximisation of the load capacity as the benchmark data, the percentage of variations in $\xi_{\text {opt }}$ and $\kappa_{\text {opt }}$ by choosing $\mu_{M}$ and $\Omega$ as the objective functions for optimisation were calculated. In this regard, Figures 10a and b demonstrate the absolute value of the percentage of variations in the optimum height and textured area ratios for different number of textures and their types/profiles.

As Figure 10a shows, excluding the particular case of $m=1$, the optimum height ratio for negative rectangular textures has the least amount of change by altering the objective function whilst both of the positive triangular shape textures have the uppermost amount of changes (with almost above 20\%) for all cases especially when minimisation of the friction coefficient is aimed. The results in this figure specify that for a given texture type and profile, optimisation based on minimisation of $\mu_{M}$ will provide higher percentage of change in $\xi_{\text {opt }}$ than based on maximisation of $\Omega$ except for negative rectangular textures. A relatively considerable amount of variation in $\xi_{\text {opt }}$ by changing the type of objective function from 
maximisation of $\Omega$ to minimisation of $\mu_{M}$ for negative triangular textures especially at higher number of textures is also considerable.

Figure 10b, which is for the variations of optimum textured area ratios, signifies, $\kappa_{\mathrm{opt}}$ for negative textures are much less sensitive to the variations in the type of objective function comparing their positive rivals. In fact, by changing the objective function from $\Lambda_{W}$ to $\mu_{M}$ or $\Omega$, the optimum textured area ratios can alternatively change from about 14 to $28 \%$ depending on the type, shape and number of textures.

\section{Figure 10}

Considering the results in Figures 10a and b, it can be realised that the optimum geometrical parameters for positive type textures can be altered more perceptible by changing the type of objective function in comparison to the negative type textures. However, a question one may ask can be that is this so for the other performance measures of the bearing. Speaking broadly, at this point, an interesting subject worth of investigating can be that how much, for instance, the load capacity of a textured bearing might drop from its maximum if the optimum geometrical parameters for a given textured bearing based on, say minimisation of friction coefficient, were employed instead. The results in Figure 10c, which are delivered for this purpose, represent the absolute value of percentage of variation (obviously reduction) in the load capacity by considering objective functions rather than the load capacity itself. These results indicate that the load capacity produced by negative textures remains very close to the maximum load capacity if even the obtained optimum geometrical parameters for the other objective functions were employed. Moreover, as the numbers of textures increase, this alteration becomes much lesser. On the other hand, the load capacity of the positive textures alters quite considerably. In fact, this is completely inline with the corresponding variations in $\xi_{\text {opt }}$ and $\kappa_{\text {opt }}$ demonstrated in Figures 10a and b. Nevertheless, it should also be noted that the amount of alteration in the load capacity is not highly substantial as in the extreme cases (i.e. for both of positive type triangular textures at higher number of textures and regardless of the type of the objective function) the reduction in the load capacity is less than $8 \%$.

\subsection{The essence of optimisation of textured slider bearings}

In this section, it is intended to reveal the significance of attempt to optimise the performance of the textured bearings. In other words, the reduction in the performance of a textured bearing by deliberate deviating of one or more of the defining geometrical parameters from their supposed optimum value(s) will be studied at this point. To do so, three scenarios were investigated. First, by setting the textured area ratio to the corresponding obtained optimum values in each studied model, the optimum height ratios were altered to be exactly, say $25 \%$ more than the corresponding optimum values. In the second scenario, by keeping the optimum height ratios at their optimum values, related textured area ratios were altered to be exactly $25 \%$ less than the corresponding optimum values. Finally, both optimum height and textured area ratios were altered to be $25 \%$ more and less than the corresponding optimum values, respectively. In all these three scenarios, using the analytical relations above, the performance of the bearing was calculated and compared to the maximum performance. This approach would also let one to signify 
the degree of influence of each geometrical parameter on the bearing performance as well as the collective influences of their alteration.

Figure 11a shows the effect of $25 \%$ increment in the textures height ratio from the corresponding optimum values on the bearing performance (considering all three defined objective functions) for all studies textures types, numbers and profiles. As is clear from this figure, the increment in the texture height ratio makes the friction coefficient to rise whilst causes the load capacity of the bearing and $\Omega$ to reduce. The amount of amplification in the friction coefficient for negative rectangular textures is the highest of all and can reach up to near $11 \%$ at higher number of textures. Meanwhile, the negative triangular textures become the least affected with about $3.6 \%$ alteration at higher number of textures. The amount of rise in the friction coefficient is to some extent higher for positive triangular textures. On the other hand, the positive rectangular ones are about 3\% (or slightly more) less affected than their negative counterparts. Considering the variations in the bearing performance based on the load capacity, from Figure 11a it can be seen that the order in which different textures appear from the most affected to the least, makes almost a very similar pattern with a slight change only in the values. However, based on the $\Omega$, a noticeable difference is the overlapping of the results for positive rectangular textures with those for positive triangular ones.

Alternatively, Figure $11 \mathrm{~b}$ is for the alterations in the bearing performances resulted in from a $25 \%$ reduction in the size of optimum textured area ratio while the optimum height ratios are preserved. According to this figure, the reduction in textured area ratio from optimum has similar effect on the bearing performance as the increment in the optimum height ratio does. It can also be seen that the order of magnitude of alteration in the bearing performance parameters are almost similar to the results shown in Figure 11a. However, considering the type of textures, clear boundaries between the results can be observed here. In fact, it can be observed that the effect of alterations in the textured area ratio is more pronounced for the negative textures. In contrast to what was already seen in the case of variations in the height ratio, the shape of textures appears to be an insignificant influential parameter especially for the negative type textures. In other words, it seems that by making a same amount of alteration in $\kappa_{\text {opt }}$, the subsequent amount of variations in the textured bearing performance would be very close to each other regardless of the shape of implemented textures especially if negative type textures were being used.

The results in Figures 11a and b compared the effects imposed on the textured bearing performance by varying either the height or textured area ratios from their optimum values separately. Figure 11c, demonstrates the collective (superimposed) effect of alterations in $\xi_{\text {opt }}$ and $\kappa_{\text {opt }}$ on the textured bearing performance. The results in this figure are obtained by $25 \%$ increasing and decreasing in the corresponding $\xi_{\text {opt }}$ and $\kappa_{\mathrm{opt}}$ values for each studied case, respectively. In this figure, it can be observed that once more, the negative textures are the most affected ones. Amongst this type of textures, the rectangular shape ones become the most affected with a distinguishable gape between the results for them and the other two triangular shape textures.

\section{Figure 11}

An interesting outcome that can be immediately emerged from comparing the results given in Figure 11 is that for the negative textures the mutual effect of alterations in $\xi_{\text {opt }}$ and $\kappa_{\text {opt }}$, as was described above, on the bearing performance, looks as if supporting each other's individual effects in altering the bearing performance in 
the same direction. For example, the friction coefficient of a bearing textured with 10 negative rectangular textures alters 10.69 and $8.40 \%$ by changing the corresponding $\xi_{\text {opt }}$ and $\kappa_{\text {opt }} 25 \%$ at a time, respectively. On the other hand, a $21.82 \%$ rise in the friction coefficient can be obtained by altering both of the optimum height and textured area ratios by $25 \%$. However, for the positive textures such a trend cannot be generalised, even if it is assumed that the individual effects are suppressing each other instead of supporting.

\subsection{The effect of variations of bearing number on the optimum values}

Up to this point, in the present study, the optimum results were revealed for bearing number of zero indicating no pressure difference at the bearing ends. In this subsection, by conducting the optimisation process for three extra bearing numbers of $0.1,0.2$ and 0.3 , it is intended to compare the optimum values as well as the textured bearings performance considering the variations of bearing number. It is noted that although the variations in the results can be studied considering all three objective functions defined above, the results based on maximisation of the dimensionless load capacity are revealed here as an example. Apparently, the same approach can be employed considering other defined performance measures for the textured bearing.

Figure 12a demonstrates the percentage of variations in the optimum height ratios for various studied texture types, numbers and profiles by employing different bearing numbers comparing to the corresponding cases where there were no pressure difference between bearing leading and trailing edges. The results in this figure indicate that increasing the bearing number elevates the optimum height ratio for the negative textures whilst it has a reverse effect on $\xi_{\text {opt }}$ for positive ones. In addition, the optimum height ratios appears to be varying quit uniformly with the bearing number for almost all of the number of textures. Furthermore, it can be seen that the effect of variations of $\Lambda$ on the optimum height ratios is lesser for positive textures. For example, by promoting $\Lambda$ from 0.1 to 0.2 and then, from 0.2 to 0.3 , corresponding to 100 and 50\% growth, respectively, the optimum height ratios for a bearing containing, say 10, negative rectangular textures vary about 100.64 and $50.45 \%$, respectively, whereas for the positive rectangular textures the equivalent values are about 87.22 and $41.42 \%$, respectively.

Figure $12 \mathrm{~b}$ illustrates the percentage of variations of the optimum textured area ratios with the number of textures for different texture types and profiles at bearing numbers of $0.1,0.2$ and 0.3 comparing to the corresponding results for $\Lambda=0$. This figure indicates much lower sensitivity of $\kappa_{\mathrm{opt}}$ to the variations in the bearing number, particularly in the case of negative textures. Except for the positive rectangular textures at bearing number of 0.3 , in which $\kappa_{\text {opt }}$ grows up to about $15.78 \%$ utmost when the maximum number of studied textures are employed, for the rest of cases, $\kappa_{\text {opt }}$ varies no more than $11 \%$. However, unlike the studied case for $\xi_{\text {opt }}$ above, where the optimum height ratios for positive textures were plummeting as the bearing number was rising; the optimum textured area ratio clearly increases with the bearing number for both positive and negative type textures.

In a similar approach to the results demonstrated in Figures 12a and b, the percentage of variations in the maximum load capacity are presented in Figure 12c. As could be expected, increasing the existing pressure difference between the bearing ends causes the maximum load capacity to boost thanks to the contribution of the hydrostatic pressure. Moreover, as the number of textures grows the rate of increment also rises. An interesting observation that can be made through the results in figure 12c is the percentage of variations for the negative textures comparing their positive 
rivals for the same amount of rise in the pressure difference. For instance, at the bearing number of 10 , by increasing the bearing number from zero to 0.2 , the maximum load capacity for positive rectangular textures enhances up to about $40.24 \%$ whilst for the negative ones a substantial amount of $153.74 \%$ augmentation can be observed.

\section{Figure 12}

\subsection{The effect of variations of area density of textures on the optimum values}

In the previous section of this study, an arbitrary area density of $S p=0.5$ was employed because the earlier tests showed that for asymmetric partially textured bearings there is no specific optimum value for area density of textures and the tribological performance of the textured bearing increases with $S p$ (or alternatively, $\varepsilon$ ). This part of the study is dedicated to observe the effect of variations of the area density of textures on the optimum values of the rest of parameters and the bearing performance. Of course, this could be conducted using any specific number of textures. However, one of the advantages of employing the analytical method is that it provides opportunity to study the conditions where using the numerical or experimental approach could encounter some difficulties. For instance, embracing the analytical relations provided in the earlier parts, it is possible to study the textured bearing performance and related optimum parameters when the number of textures becomes enormous or in fact, mathematically infinite. In the case of the asymmetric partially textured bearings, this would be very alike to study the effect of the concept of inlet roughening introduced by Tonder [28, 29]. For this purpose, the limit of the related equations for the bearing performance was obtained for when the number of textures was approaching to infinite and then, the variations of optimum height and textured area ratios were obtained by shifting the area density of textures. Although the approach could be used considering any of the given performance parameters defined before and at any specified bearing number, the results for the case of maximisation of dimensionless load capacity and $\Lambda=0$ are presented here for instance.

According to Figure 13a, which is for the variations of the optimum height ratio with the area density of textures, $\xi_{\text {opt }}$ for negative textures increases with $S p$ in higher rates at higher area densities. On the other hand, for positive textures, $\xi_{\text {opt }}$ reduces by increasing $S p$ and this reduction has much higher rates at lower area densities. As it could be expected, since $m \rightarrow \infty$ therefore, the results for both rectangle- and isosceles-triangular textures are overlapped. It can also be detected that at the very low area densities, the positive textures demand very high optimum height ratios. In addition, apart from negative rectangular textures, the other texture types and profiles seem to have very close optimum height ratios at area density of about 0.5 .

Similar to the variations of $\xi_{\text {opt }}$ with $S p$, the optimum textured area ratios (see Figure 13b) for negative and positive textures have ascending and descending trends with $S p$, respectively. In other words, by increasing the area density of textures, the size of the optimum textured area for positive textures shrinks while for the negative ones it expands. Another interesting point is that for the negative textures, the shape of the textures in terms of being rectangular or triangular appears to have not much significant effect on $\kappa_{\text {opt }}$ in the first half of variations of $S p$ whereas for the positive textures a noticeable gap separates the results for rectangular and triangular textures.

The variations of maximum dimensionless load capacity with $S p$ for different texture types and profiles are demonstrated in Figure 13c. According to Figure 13c, 
although for both negative and positive textures $\left(\Lambda_{W}\right)_{\max }$ increases with $S p$, this increment happens in a quite different manner. In fact, for the negative textures, the rate of amplification in $\left(\Lambda_{W}\right)_{\max }$ increases with $S p$ whilst for the positive ones, the rate of grow increases as $S p$ decreases. In addition, the effect of the shape of the textures of the negative type in terms being triangular or rectangular becomes more pronounced at higher $S p$ values.

\section{Figure 13}

In addition to the result above, Tables 1 and 2 list the optimum values for height ratios and textured portions at $S p=0.5$ corresponding to the maximisation or minimisation of the related objective functions when the number of textures becomes infinite for the studied texture types and profiles. The resultant ultimate values for the optimised functions are also listed in these tables. As is seen, the rectangle- and isosceles-triangular textures both have identical optimum values either in negative or positive forms. In addition, the rectangular textures show much better performances in terms of all of the considered objective functions in both negative and positive texture types.

\section{Table 1}

\section{Table 2}

\section{Conclusions}

An analytical model for analysis of the slider bearings with textured surfaces was developed. The obtained analytical relations provided an excellent sight into the way the bearing performance parameters are connected to and altered by the variations of the affecting dimensionless parameters. Three parameters to describe the performance of a textured bearing were introduced including the load capacity, friction coefficient and load capacity to lubricant flow rate ratio. The nature of the obtained equations suggested employing a sophisticated optimisation approach. Therefore, the optimisations were conducted using a non-linear novel search/optimisation method based on evolutionary algorithms. The importance of optimisation based on various objective functions as well as the significance of optimisation for textured bearings were highlighted. In addition, the effect of variations of the bearing number and the area density of the textures on the optimum textured bearing configuration as well as the textured bearing performance were studied in details. It was shown that by employing the analytical approach it is possible to study the textured surfaces at extreme such as when $m \rightarrow \infty$. Moreover, the dimensionless form of the analytical relations and the obtained results from the optimisation process enables one to apply those in any scale as long as the fundamental assumptions, which Reynolds lubrication equation is based on, are not violated. In other words, by proper adjusting of the geometrical parameters the approach allows one to study both 'macro-wedge' and 'micro-wedge' effects. In the former a few coarse textures are employed whilst the latter case can contain numerous fine-sized textures.

It was shown that although introducing surface texturing on a slider bearing with smooth parallel flat surfaces can produce substantial amount of load capacity, comparing in the optimum configurations, increasing the number of textures would improve none of the defined performance measures for the textured bearing in pure 
hydrodynamic lubrication regime. For the area density of textures, it was shown that the maximum possible value is the most favourable.

In general, the following results from the present study can be concluded: 1. The optimum bearing parameters based on maximising of load carrying capacity vary with those obtained and are based on minimisation of the friction coefficient or maximisation of load capacity to lubricant flow rate ratio, particularly when the number of textures is smaller.

2. The shape and type of textures can be very important parameters on affecting the performance of a textured bearing.

3. For all performance measures, the positive rectangular textures provided the best results with remarkable distance from the others.

4. The effect of the shape and type of textures on the optimum results is more pronounced when $\mu_{M}$ and $\Omega$ are considered as the objective functions for optimisation.

5 . The shape of textures can affect the trend of variations of optimum geometrical parameters with increasing the number of textures.

6. For rectangle- and isosceles-triangular shape textures, the difference in the corresponding optimum height and textured area ratios as well as the performance of the textures can be distinguished at lower number of textures. In addition, at lower number of textures, the difference between the results for isosceles- and rectangletriangular shape textures of the same type is more noticeable for positive textures. At higher number of textures, the results for these two usually overlap.

7. The sensitivity of optimum height ratio to textures geometry is higher than that of the textured area ratio especially when the number of textures increases.

8. Based on minimisation of $\mu_{M}$, the positive textures in any studied profile outperform the negative ones.

9. Based on maximisation of either $\Lambda_{W}$ or $\Omega$, the rectangular textures of any type perform better than triangular ones.

10. In general and particularly for higher number of textures, the negative rectangular textures demand the lowest $\xi_{\text {opt }}$, whilst the positive rectangular ones require the least $\kappa_{\text {opt }}$ values. In addition, the positive type textures in any profile have lesser $\kappa_{\text {opt }}$ than the positive ones.

11. Apart from a few number of textures in the start, $\kappa_{\text {opt }}$ for all negative textures become very close to each other regardless of the shape of textures. On the other hand, the effect of the shape of textures is more recognisable for positive textures. 12. As the number of textures grows, the rate of variations of $\xi_{\mathrm{opt}}$ and $\kappa_{\mathrm{opt}}$ diminishes. 13. By employing the optimum values obtained based on minimisation of $\mu_{M}$ or maximisation of $\Omega$, the load capacity for positive isosceles- and rectangle-triangular textures drops mores than the others.

14. It was shown that by failing to employ the optimum values for height and textured area ratios; the performance of the bearing textured with negative rectangular textures reduces more than the other textures.

15. By increasing the pressure difference between the bearing ends, the optimum height ratios for negative and positive textures almost uniformly increase and reduce, respectively. At the same time, the optimum textured area ratios and the maximum dimensionless load capacities increase for all studied texture shape/types.

16. By increasing $S p, \xi_{\text {opt }}$ and $\kappa_{\text {opt }}$ for negative textures increase whilst for the positive textures reduce. In addition, by increasing $S p,\left(\Lambda_{W}\right)_{\max }$ for both of the positive and negative type textures increases with different rates of increments.

A further interesting outcome of this study is that the results can be considered as a good evaluation tool for hydrodynamic effects and tribological performance of 
tribodevices employing 'inlet roughness' concept, those described by Tonder [28, 29] when the number of textures becomes numerous.

\section{References}

[1] Erdemir A. Review of engineered tribological interfaces for improved boundary lubrication. Tribol. Int. 2005; 38: 249-256

[2] Etsion I., Kligerman Y., Halperin G. Analytical and experimental investigation of laser-textured mechanical seal faces. Tribol. Trans. 1999; 42 (3): 511-516

[3] Ronen A., Etsion I., Kligerman Y. Friction-reducing surface-texturing in reciprocating automotive components. Tribol. Trans. 2001; 44 (3): 359-366

[4] Ryk G., Kligerman Y., Etsion I. Experimental investigation of laser surface texturing for reciprocating automotive components. Tribol. Trans. 2002; 45 (4): 444449

[5] Rahmani, R. An investigation into analysis and optimisation of textured slider bearings with application in piston-ring/cylinder liner contact. PhD Thesis, Anglia Ruskin University, UK; 2009

[6] Pettersson U., Jacobson S. Textured surfaces for improved lubrication at high pressure and low sliding speed of roller/piston in hydraulic motors. Tribol. Int. 2007; 40: 355-359

[7] Salama M.E. The effect of macro-roughness on the performance of parallel thrust bearings. Proc. Inst. Mech. Eng. London 1952; 163: 149-158

[8] Hamilton D.B., Walowit J.A., Allen, C.M. A theory of lubrication by microirregularities. ASME Trans. J. Basic Engineering 1966; 88: 177-185

[9] Anno J.N., Walowit J.A., Allen, C.M. Microasperity lubrication. ASME Trans. J. Lubr. Tech. 1968; 90: 351-355

[10] Anno J.N., Walowit J.A., Allen C.M. Load support and leakage from microasperity-lubricated face seals. ASME Trans. J. Lubr. Tech. 1969; 91: 726-731 [11] Etsion I., Burstein L. A model for mechanical seals with regular microsurface structure. Tribol. Trans. 1996; 39 (3): 677-683

[12] Etsion I., Kligerman Y., Halperin G. Analytical and experimental investigation of laser-textured mechanical seal faces. Tribol. Trans. 1999; 42 (3): 511-516

[13] Siripuram R.B. Analysis of hydrodynamic effects of microasperity shapes on thrust bearing surfaces. Master Thesis, University of Kentucky, US; 2003

[14] Razzaque M.M., Faisal, M.T.R. Performance of mechanical face seals with surface micropores. Technical Brief, Journal of Mechanical Engineering, Transaction of the Mechanical Engineering Division, The Institution of Engineers, Bangladesh 2007; ME37: 77-80

[15] Brizmer V., Kligerman Y., Etsion, I. A laser surface textured parallel thrust bearing. Tribol. Trans. 2003; 46 (3): 397-403

[16] Sahlin F. CFD-analysis of hydrodynamic lubrication of textured surfaces. Master Thesis, Lulea University of Technology, Sweden; 2003

[17] Sahlin F., Almqvist A., Glavatskih S., Larsson R. Two-dimensional CFDanalysis of micro-patterned surfaces in hydrodynamic lubrication. J. Tribol. 2005; 127: 96-102

[18] Cupillard S. Lubrication of conformal contacts with surface texturing. Licentiate Thesis, Lulea University of Technology, Sweden; 2007

[19] Huynh B.P., Loe S. Influence of location, number and shape of corrugations in slider bearings. ANZIAM J. 2004; 45 (E): C1017-C1038 
[20] Pascovici M., Marian V., Gaman, D. Analytical and numerical approach of load carrying capacity for partially textured slider. Proc. Int. Nanotribology Conf., Nano Sikkim II: Friction and Biotribology, Nov. 8-12, Peeling, Sikkim, India; 2004

[21] Pascovici M.D., Cicone T., Fillon M., Dobrica M.B. Analytical investigation of a partially textured parallel slider. Proc. IMechE, Part J: J. Eng. Tribol. 2008; 223: 151158

[22] Wong V., Tian T., Moughon L., Takata R., Jocsak J., Stanglmaier R., et al. Lowengine-friction technology for advanced natural-gas reciprocating engines. Annual Technical Progress Report for Apr. 2005 to May 2006, Massachusetts Institute of Technology (MIT), submitted to Department of Energy of National Energy Technology Laboratory, June 2006, US

[23] Wang X., Adachi K., Otsuka K., Kato K. Optimization of the surface texture for silicon carbide sliding in water. Appl. Surf. Sci. 2006; 253: 1282-1286

[24] Etsion I. (2005) State of the art in laser surface texturing. ASME J. Tribol. 2005; 127: $248-253$

[25] Rahmani R., Shirvani A., Shirvani H. Optimization of partially textured parallel thrust bearings with square-shaped micro-dimples. STLE Tribol. Trans. 2007; 50 (3): 401-406

[26] Pinkus O., Strenlicht B. Theory of hydrodynamic lubrication. McGraw-Hill Inc., US; 1961

[27] Rahmani R., Shirvani A., Shirvani H. Analytical analysis and optimisation of the Rayleigh step slider bearing. Tribol. Int. 2009; 42: 666-674

[28] Tonder K. Inlet roughness tribodevices: dynamic coefficients and leakage.

Tribol. Int. 2001; 34: 847-852

[29] Tonder K. Hydrodynamic effects of tailored inlet roughnesses: extended theory.

Tribol. Int. 2004; 37: 137-142 
Tribology International, 2010, Vol. 43, No. 8, pp. 1551-1565

(Accepted Version)

\section{List of Tables}

Table 1 . The optimum values providing the maximum $\Lambda_{W}$ and $\Omega$ as well as minimum $\eta_{M}$ for various negative textures at $m \rightarrow \infty$ in an asymmetrical partially textured bearing with $S p=0.5$

Table 2. The optimum values providing the maximum $\Lambda_{W}$ and $\Omega$ as well as minimum $\eta_{\mathrm{M}}$ for various positive textures at $m \rightarrow \infty$ in an asymmetrical partially textured bearing with $S p=0.5$ 


\section{List of Figures}

Figure 1. Schematic of a textured surface in general form

Figure 2. The rectangular textures and textured bearing geometry

Figure 3. Schematic of a slider bearing with negative (a) isosceles-triangular and (b) rectangle-triangular shape textures

Figure 4. Schematic of a slider bearing with positive (a) rectangular, (b) isoscelestriangular and (c) rectangle-triangular shape textures

Figure 5. Dimensionless pressure distributions for different tested texturing patterns: (a) Series I and (b) Series II

Figure 6. Variations of optimum (a) height and (b) textured area ratios as well as (c) maximum dimensionless load capacities with the number of textures for different studied texture types and profiles

Figure 7. Variations of optimum (a) height and (b) textured area ratios as well as (c) minimum modified friction coefficient with the number of textures for different studied texture types and profiles

Figure 8. Variations of optimum (a) height and (b) textured area ratios as well as (c) maximum load capacity per lubricant flow rate versus the number of textures for different studied texture types and profiles

Figure 9. Dimensionless pressure distributions for (a) various texture types and shapes at $m=5$ and (b) isosceles-triangular textures at $m=1$ and 2

Figure 10. Variations of optimum (a) height and (b) textured area ratios by changing the type of objective function as well as (c) variations in the corresponding load capacities comparing the maximum possible load capacity ${ }^{7}$

Figure 11. Percentage of variations (reductions) in the bearing performances by implementing of $25 \%$ (a) increase in optimum height ratios, (b) decrease in optimum textured area ratios and (c) increase and decrease in both height and textured area ratios, respectively

Figure 12. Percentage of variations of optimum (a) height and (b) textured area ratios as well as (c) maximum dimensionless load capacity versus the number of textures for different studied texture types and profiles at different bearing numbers

\footnotetext{
${ }^{7}$ The parameters inside the parentheses represent the type of objective function.
} 
Tribology International, 2010, Vol. 43, No. 8, pp. 1551-1565

(Accepted Version)

Figure 13. Percentage of variations of optimum (a) height and (b) textured area ratios as well as (c) maximum dimensionless load capacity with area density of textures for different studied texture types and profiles 
Tribology International, 2010, Vol. 43, No. 8, pp. 1551-1565

(Accepted Version)

Table 1

\begin{tabular}{|c|c|c|c|c|c|c|c|c|c|}
\hline \multirow{2}{*}{$\begin{array}{c}\boldsymbol{m} \rightarrow \infty \\
\boldsymbol{S p}=\mathbf{0 . 5}\end{array}$} & \multicolumn{3}{|c|}{$\begin{array}{c}\text { Based on maximisation of } \\
\text { dimensionless load } \\
\text { capacity }\end{array}$} & \multicolumn{4}{c|}{$\begin{array}{c}\text { Based on minimisation of } \\
\text { modified friction coefficient }\end{array}$} & \multicolumn{2}{c|}{$\begin{array}{c}\text { Based on maximisation of } \\
\text { dimensionless load } \\
\text { capacity to lubricant flow } \\
\text { rate ratio }\end{array}$} \\
\cline { 2 - 11 } & $\xi_{\mathrm{opt}}$ & $\kappa_{\mathrm{opt}}$ & $\left(\Lambda_{\mathrm{W}}\right)_{\max }$ & $\xi_{\mathrm{opt}}$ & $\kappa_{\mathrm{opt}}$ & $\left(\mu_{\mathrm{M}}\right)_{\min }$ & $\xi_{\mathrm{opt}}$ & $\kappa_{\mathrm{opt}}$ & $\Omega_{\mathrm{max}}$ \\
\hline Rectangular & 1.5777 & 0.5580 & 0.0687 & 1.5796 & 0.5686 & 14.0029 & 1.5753 & 0.5442 & 0.1308 \\
\hline $\begin{array}{c}\text { Rectangle- } \\
\text { Triangular }\end{array}$ & 2.1746 & 0.5503 & 0.0564 & 2.1989 & 0.5598 & 17.1226 & 2.1701 & 0.5392 & 0.1084 \\
\hline $\begin{array}{c}\text { Isosceles- } \\
\text { Triangular }\end{array}$ & 2.1746 & 0.5503 & 0.0564 & 2.1989 & 0.5598 & 17.1226 & 2.1701 & 0.5392 & 0.1084 \\
\hline
\end{tabular}


Tribology International, 2010, Vol. 43, No. 8, pp. 1551-1565

(Accepted Version)

Table 2

\begin{tabular}{|c|c|c|c|c|c|c|c|c|c|}
\hline \multirow{2}{*}{$\begin{array}{c}\boldsymbol{m} \rightarrow \infty \\
S p=0.5\end{array}$} & \multicolumn{3}{|c|}{$\begin{array}{c}\text { Based on maximization of } \\
\text { dimensionless load } \\
\text { capacity }\end{array}$} & \multicolumn{3}{c|}{$\begin{array}{c}\text { Based on minimisation of } \\
\text { modified friction coefficient }\end{array}$} & \multicolumn{2}{c|}{$\begin{array}{c}\text { Based on maximisation of } \\
\text { dimensionless load } \\
\text { capacity to lubricant flow } \\
\text { rate ratio }\end{array}$} \\
\cline { 2 - 11 } & $\xi_{\mathrm{opt}}$ & $\kappa_{\mathrm{opt}}$ & $\left(\Lambda_{\mathrm{W}}\right)_{\max }$ & $\xi_{\mathrm{opt}}$ & $\kappa_{\mathrm{opt}}$ & $\left(\mu_{\mathrm{M}}\right)_{\min }$ & $\xi_{\mathrm{opt}}$ & $\kappa_{\mathrm{opt}}$ & $\Omega_{\mathrm{max}}$ \\
\hline Rectangular & 2.1214 & 0.3034 & 0.1548 & 2.2966 & 0.2127 & 4.7910 & $\begin{array}{c}2.014 \\
9\end{array}$ & 0.3860 & 0.2252 \\
\hline $\begin{array}{c}\text { Rectangle- } \\
\text { Triangular }\end{array}$ & 2.2470 & 0.3961 & 0.0653 & 2.9310 & 0.2864 & 8.2692 & $\begin{array}{c}1.744 \\
6\end{array}$ & 0.4605 & 0.0778 \\
\hline $\begin{array}{c}\text { Isosceles- } \\
\text { Triangular }\end{array}$ & 2.2470 & 0.3961 & 0.0653 & 2.9310 & 0.2864 & 8.2692 & $\begin{array}{c}1.744 \\
6\end{array}$ & 0.4605 & 0.0778 \\
\hline
\end{tabular}


Tribology International, 2010, Vol. 43, No. 8, pp. 1551-1565

(Accepted Version)

Figure 1

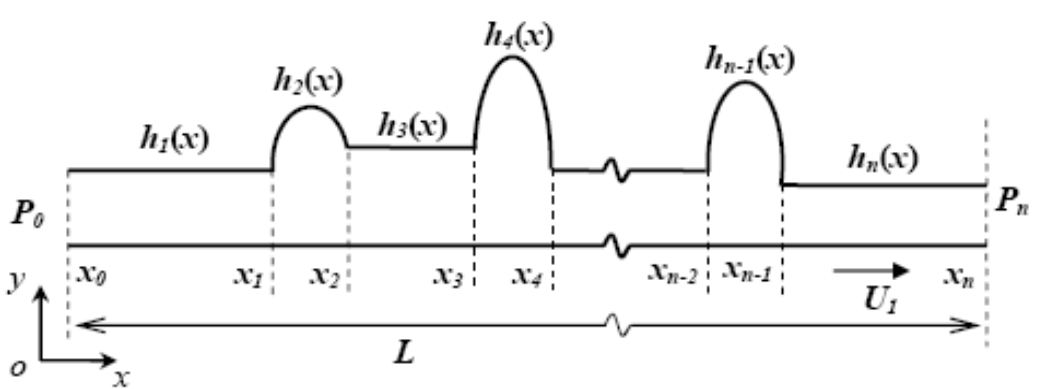


Tribology International, 2010, Vol. 43, No. 8, pp. 1551-1565

(Accepted Version)

Figure 2

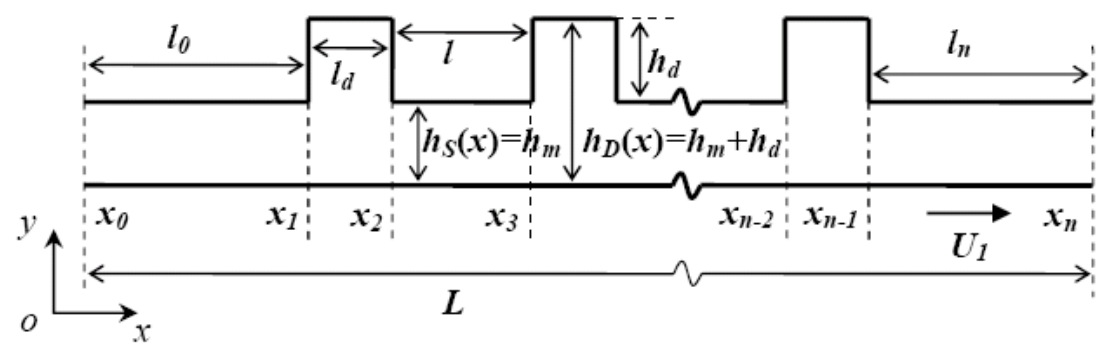


Tribology International, 2010, Vol. 43, No. 8, pp. 1551-1565

(Accepted Version)

Figure 3

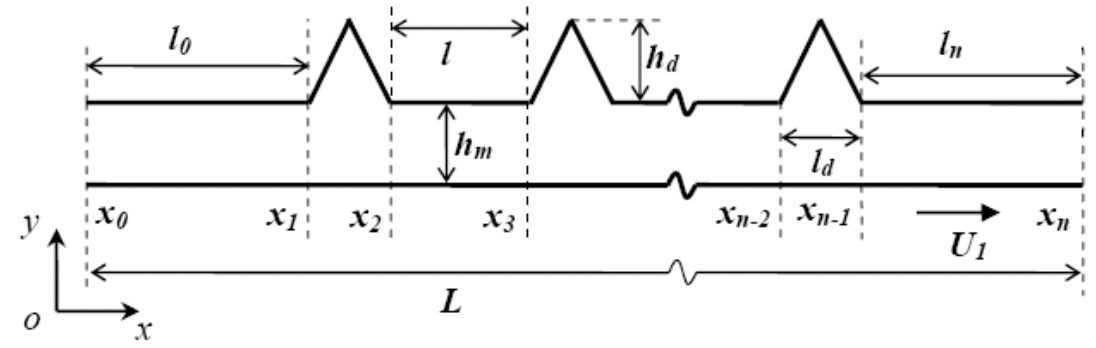

(a)

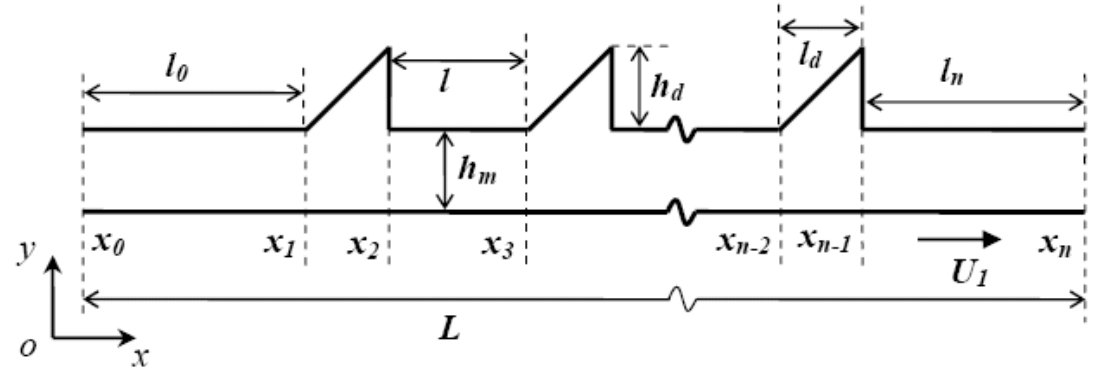

(b) 
Tribology International, 2010, Vol. 43, No. 8, pp. 1551-1565

(Accepted Version)

Figure 4

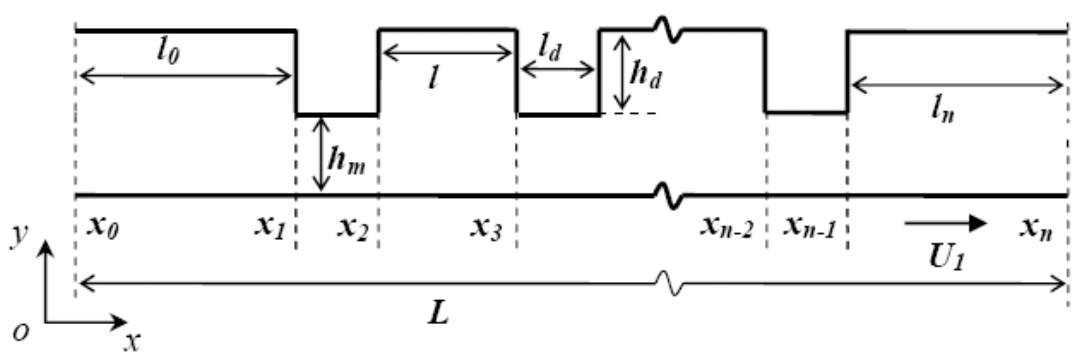

(a)

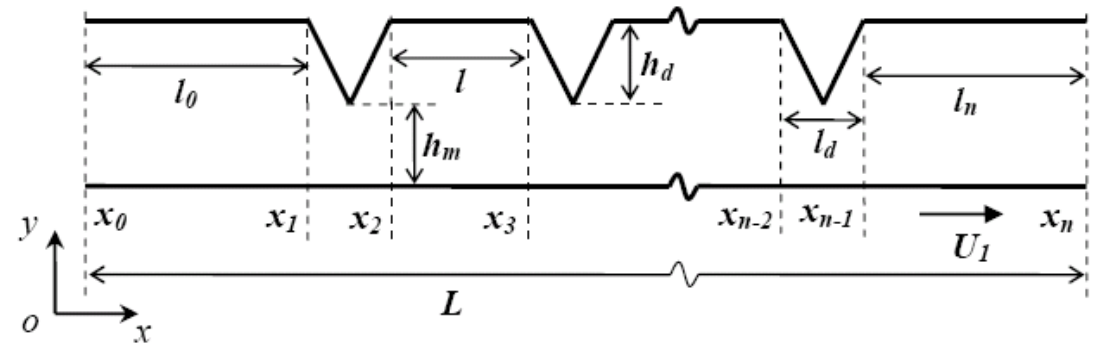

(b)

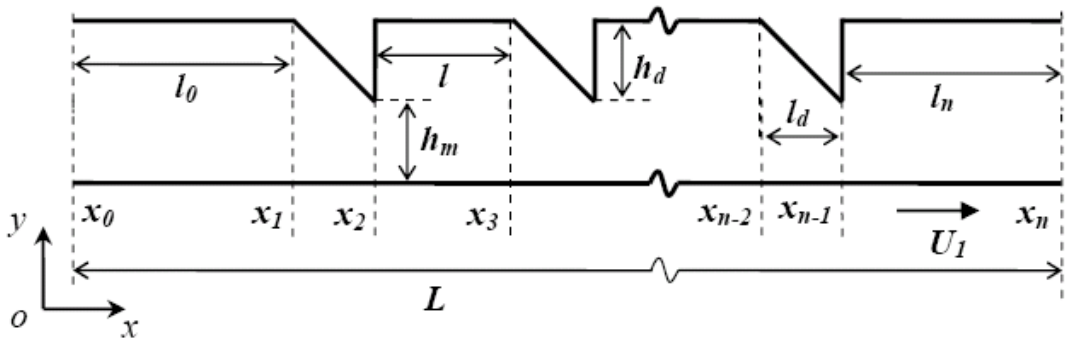

(c) 
Tribology International, 2010, Vol. 43, No. 8, pp. 1551-1565

(Accepted Version)

Figure 5

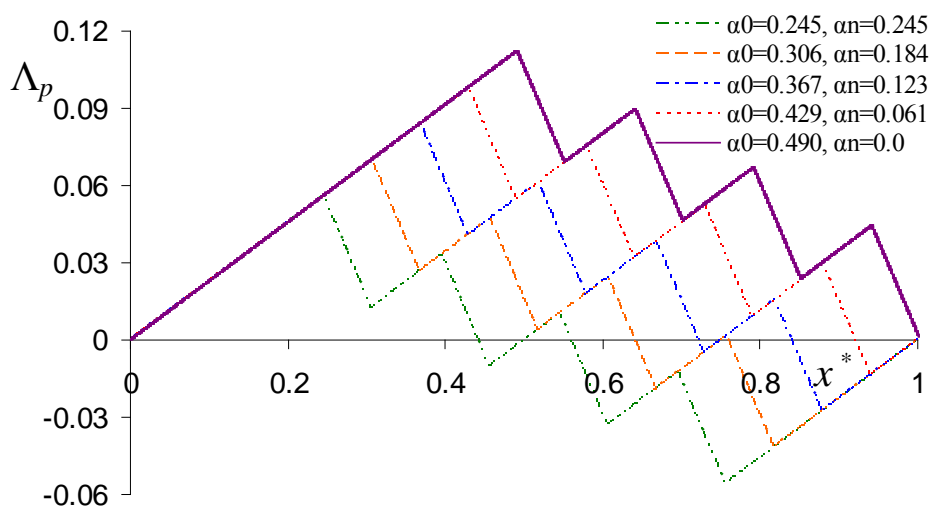

(a)

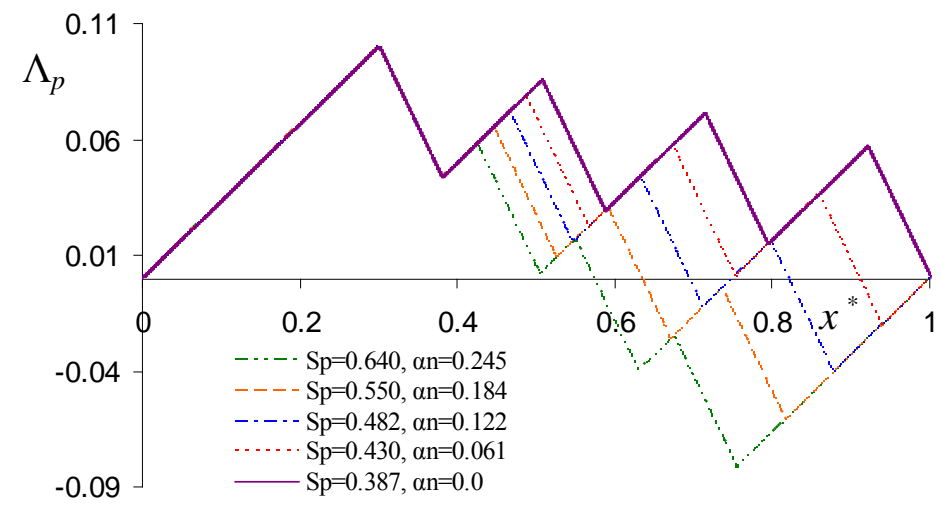

(b) 
Tribology International, 2010, Vol. 43, No. 8, pp. 1551-1565

(Accepted Version)

Figure 6

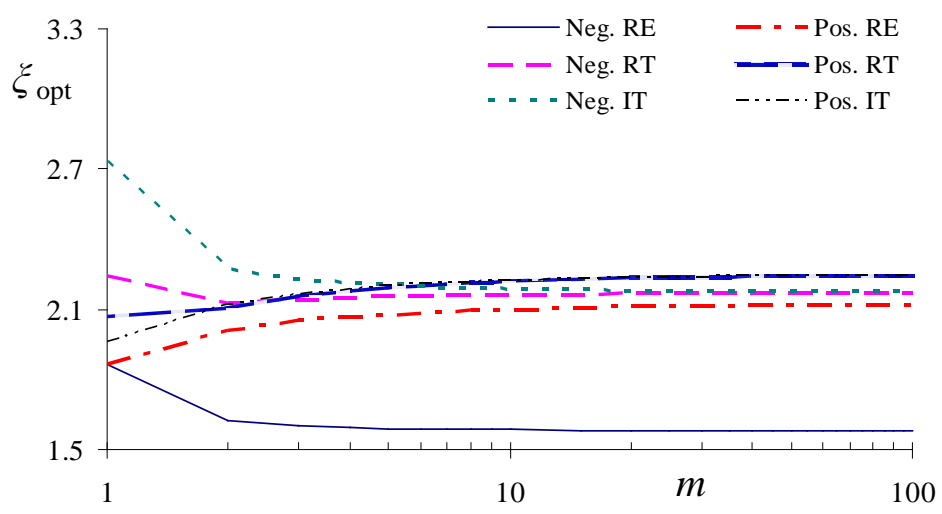

(a)

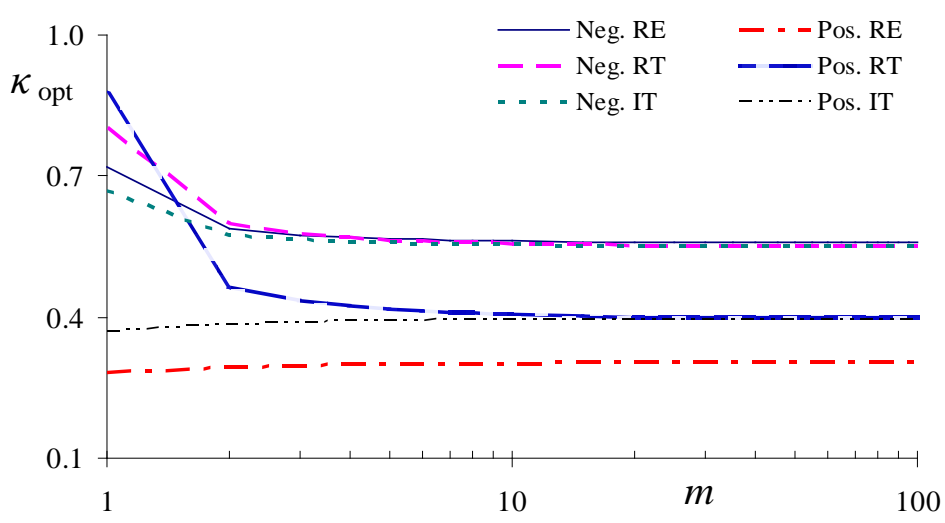

(b)

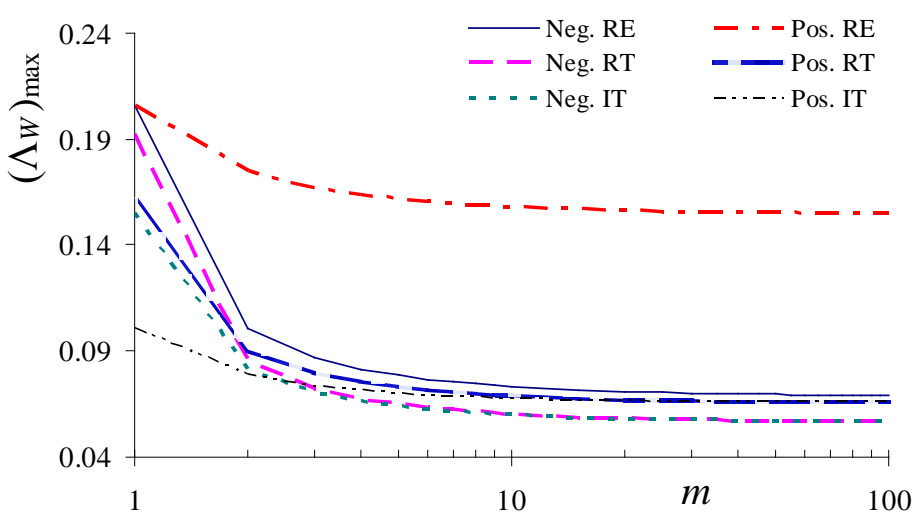

(c) 
Tribology International, 2010, Vol. 43, No. 8, pp. 1551-1565

(Accepted Version)

Figure 7

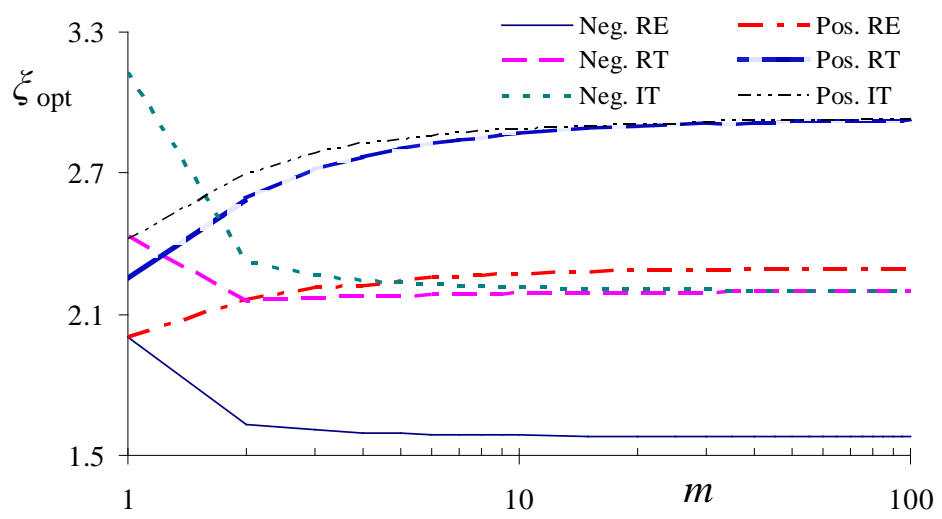

(a)

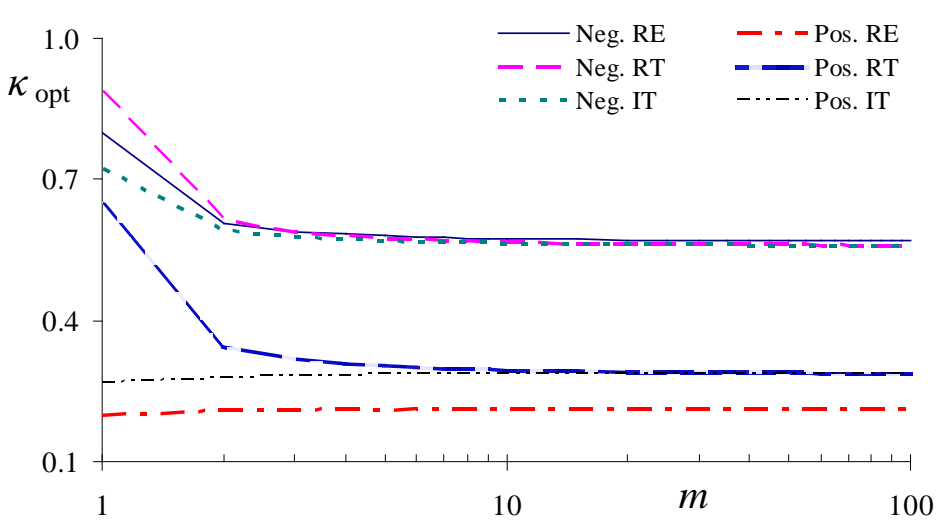

(b)

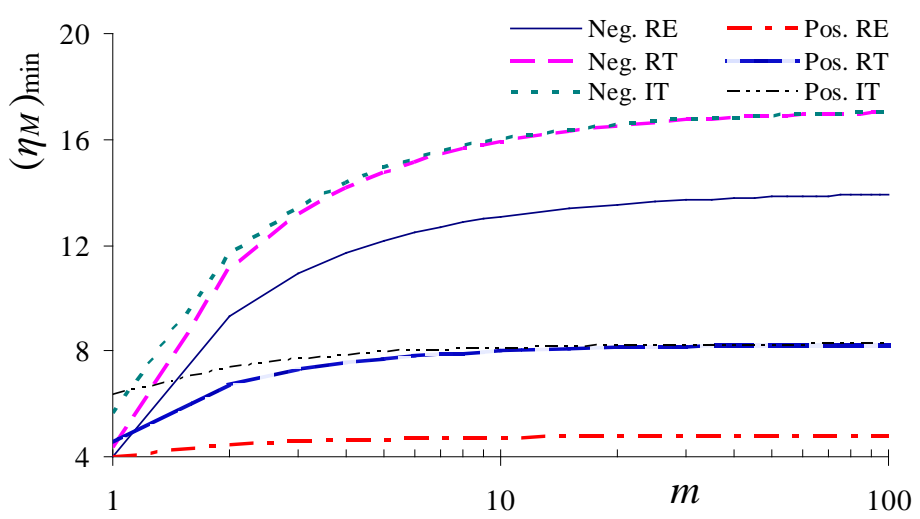

(c) 
Tribology International, 2010, Vol. 43, No. 8, pp. 1551-1565

(Accepted Version)

Figure 8

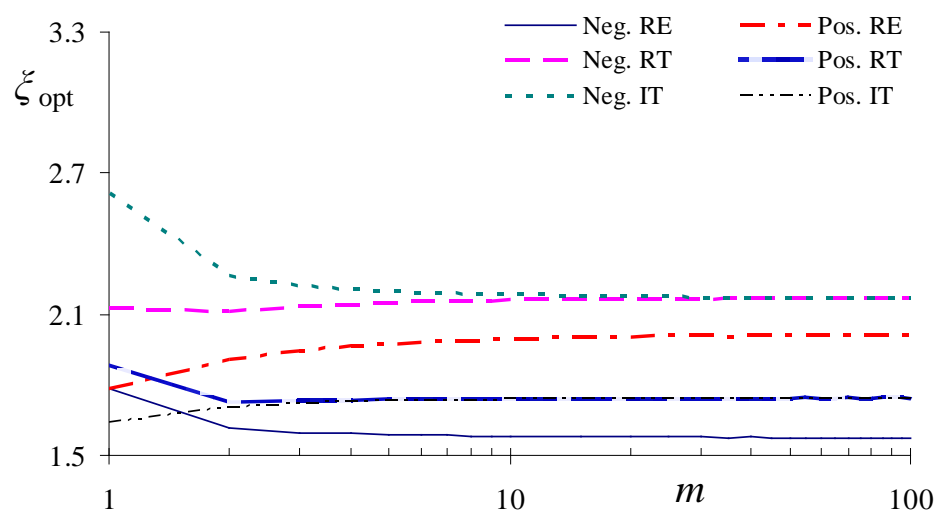

(a)

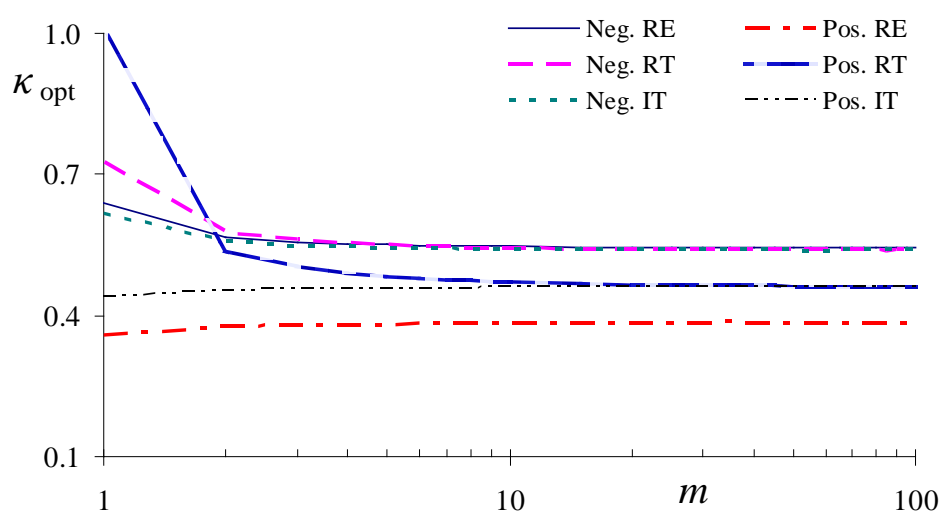

(b)

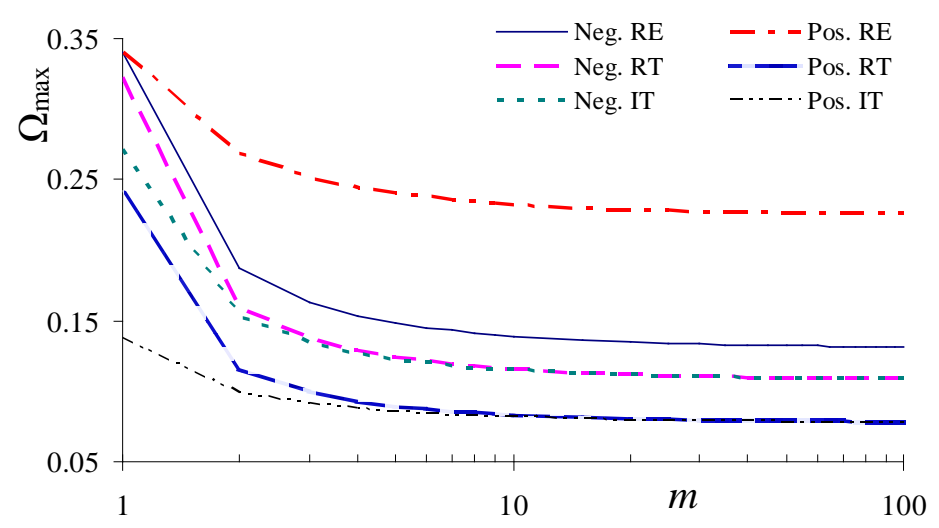

(c) 
Tribology International, 2010, Vol. 43, No. 8, pp. 1551-1565

(Accepted Version)

Figure 9

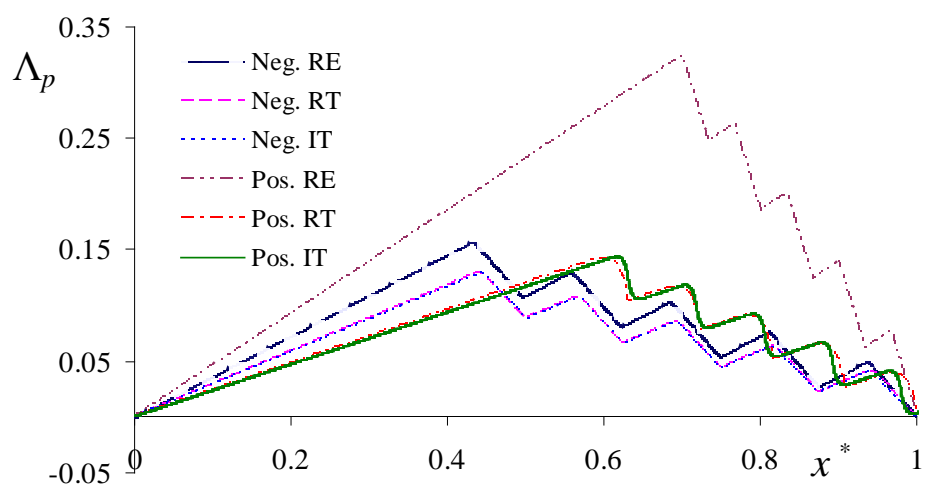

(a)

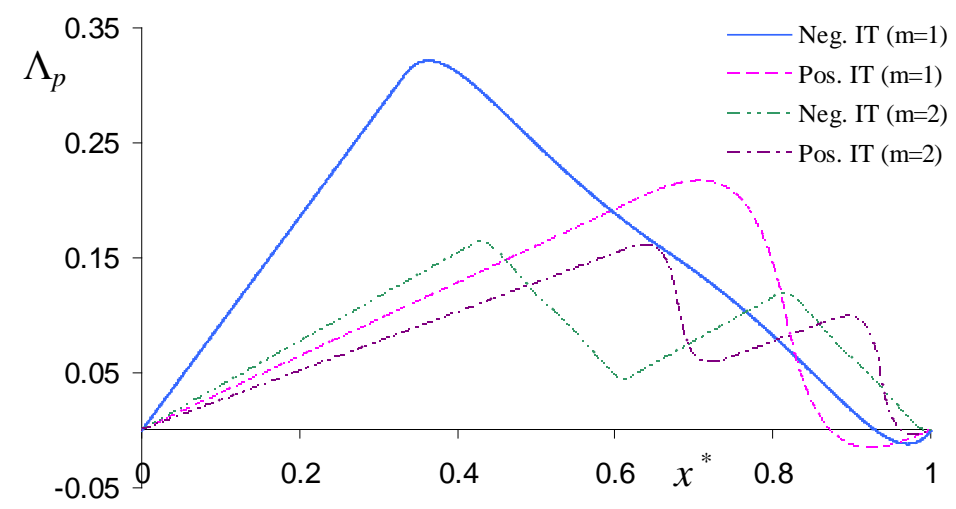

(b) 
Tribology International, 2010, Vol. 43, No. 8, pp. 1551-1565

(Accepted Version)

Figure 10

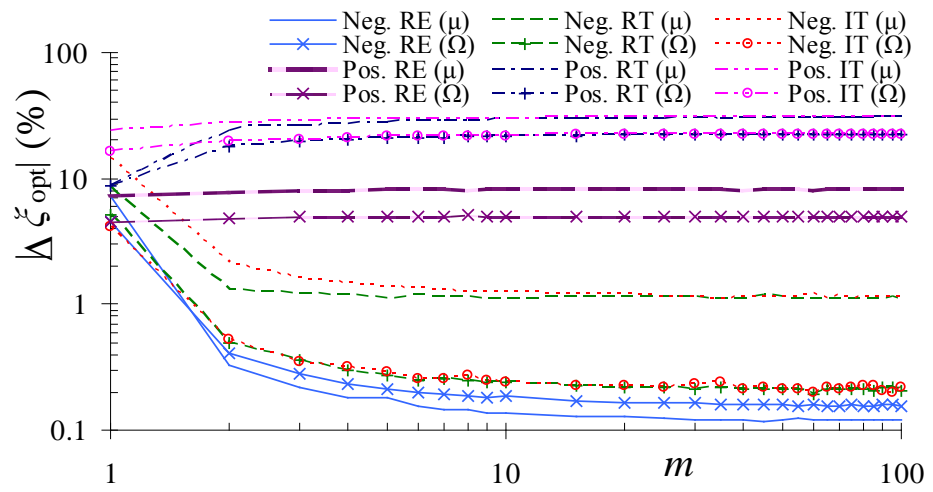

(a)

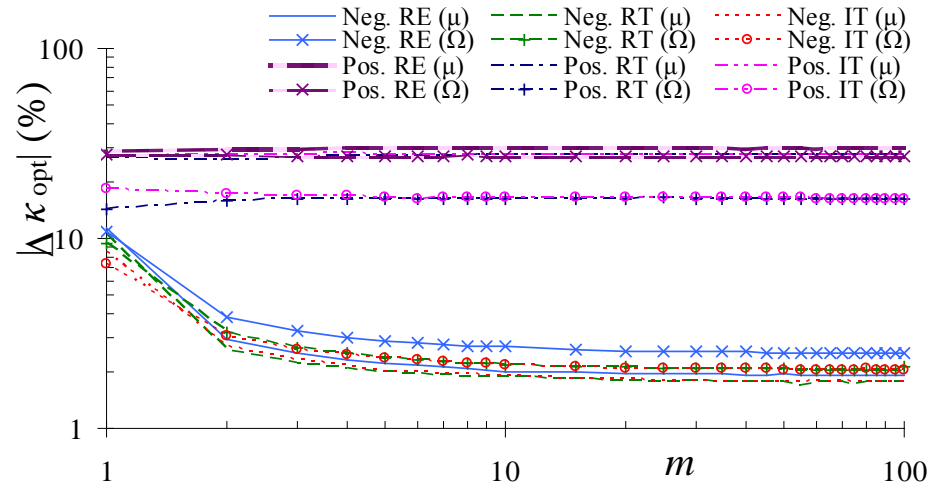

(b)

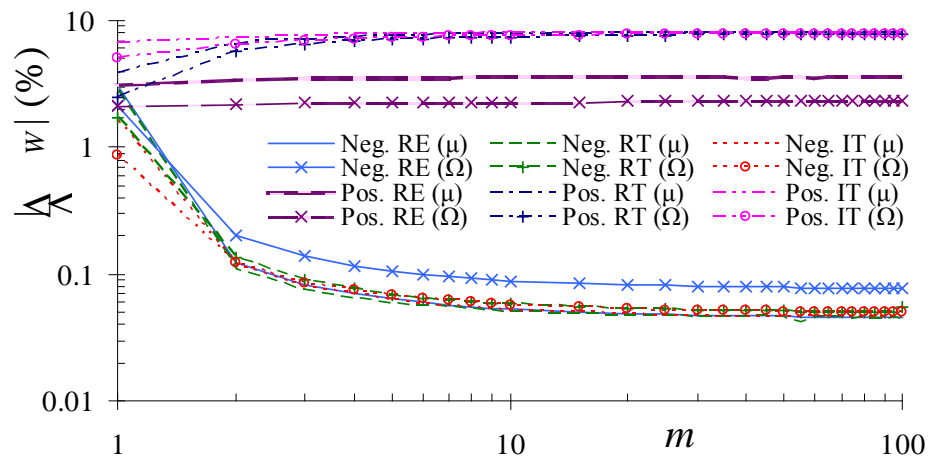

(c) 
Tribology International, 2010, Vol. 43, No. 8, pp. 1551-1565

(Accepted Version)

Figure 11

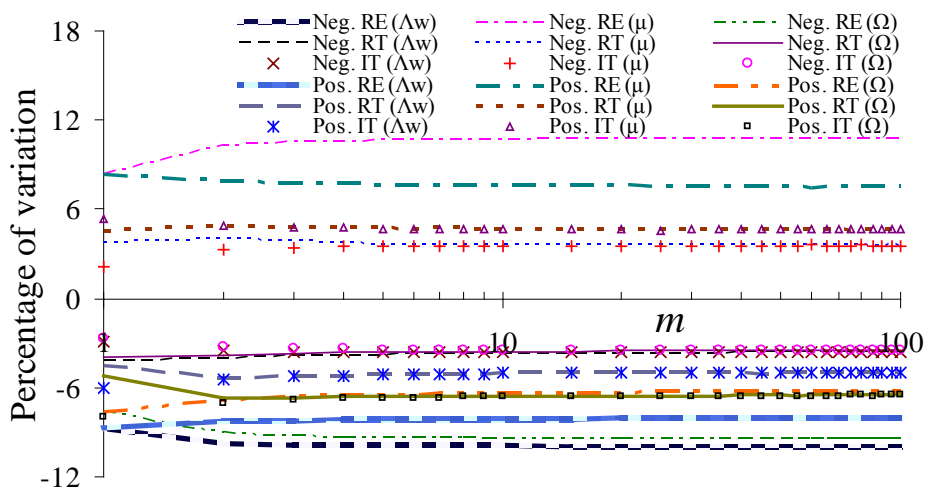

(a)

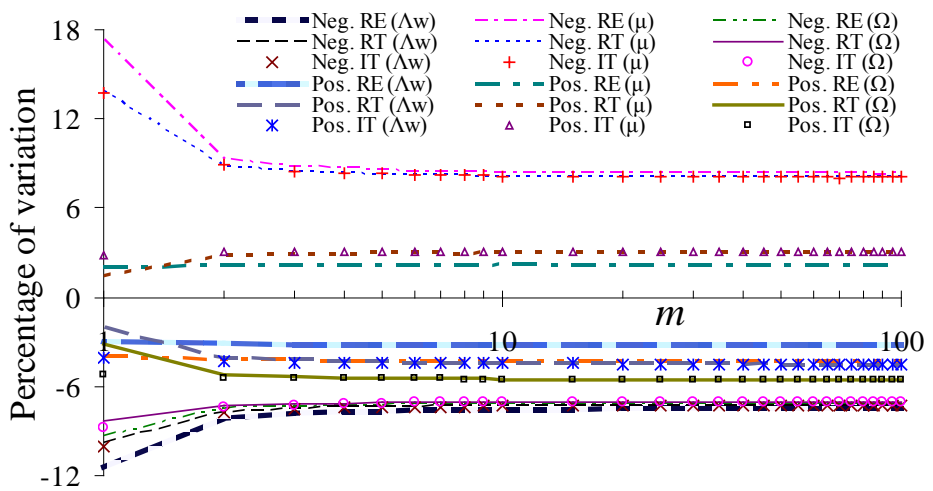

(b)

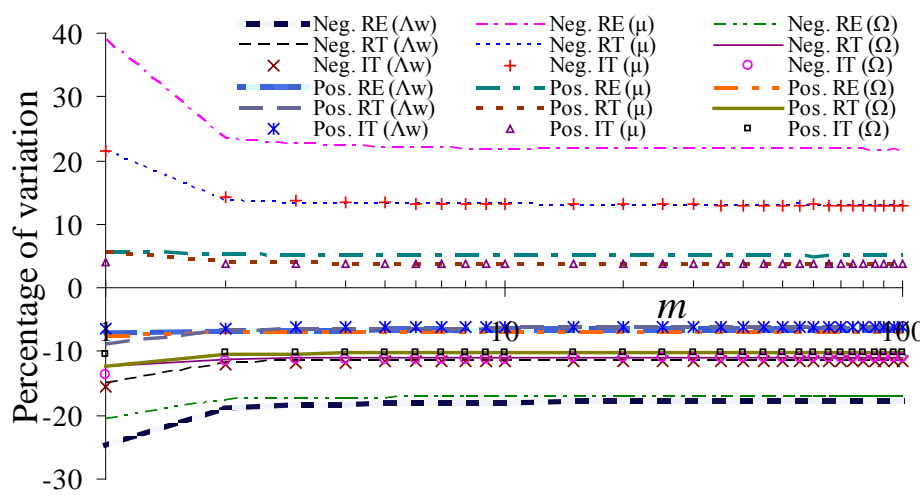

(c) 
Tribology International, 2010, Vol. 43, No. 8, pp. 1551-1565

(Accepted Version)

Figure 12

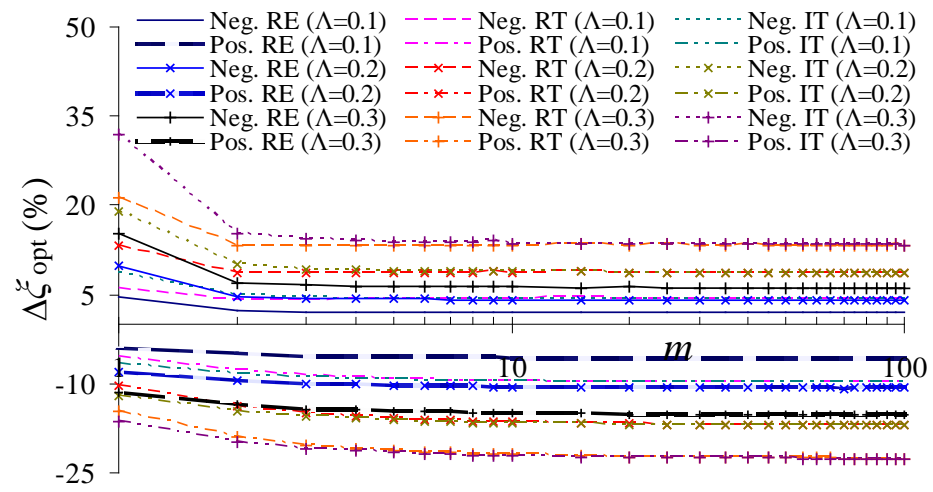

(a)

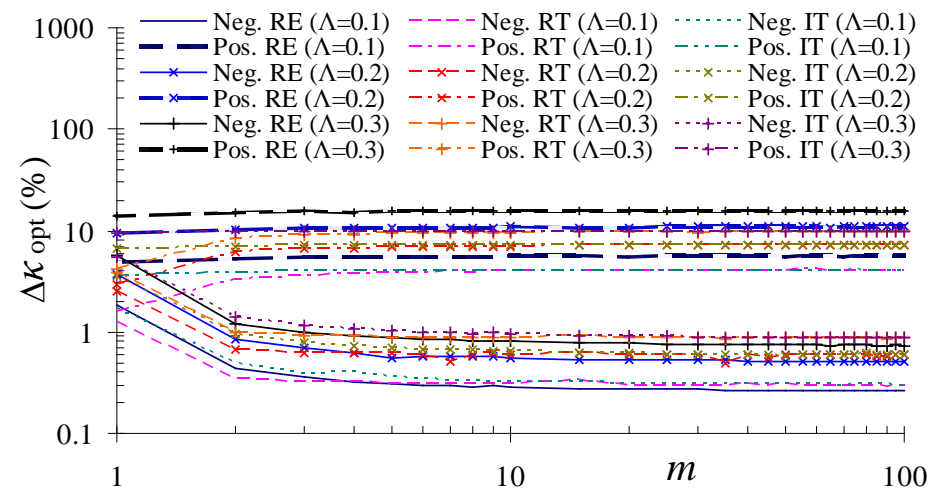

(b)

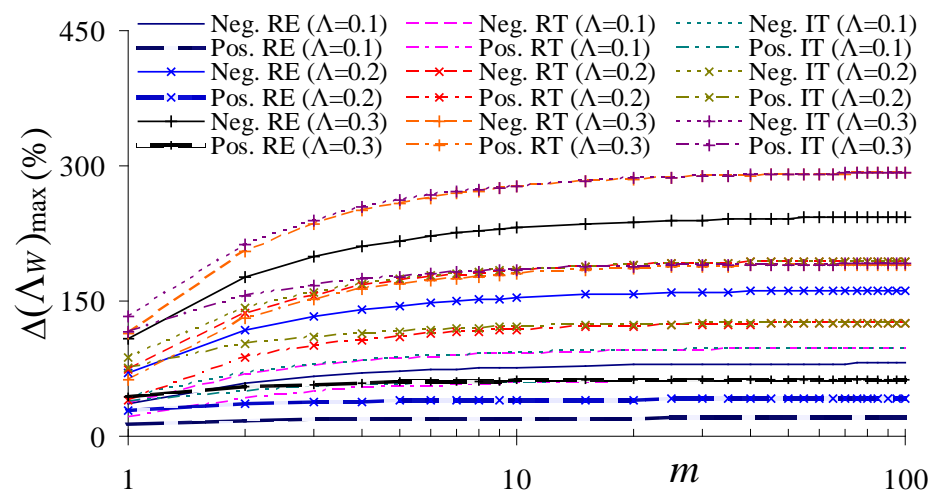

(c) 
Tribology International, 2010, Vol. 43, No. 8, pp. 1551-1565

(Accepted Version)

Figure 13

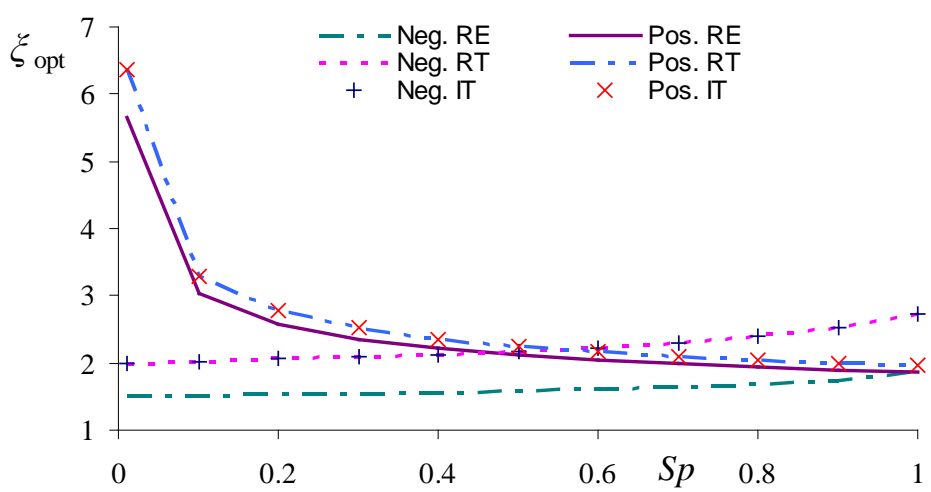

(a)

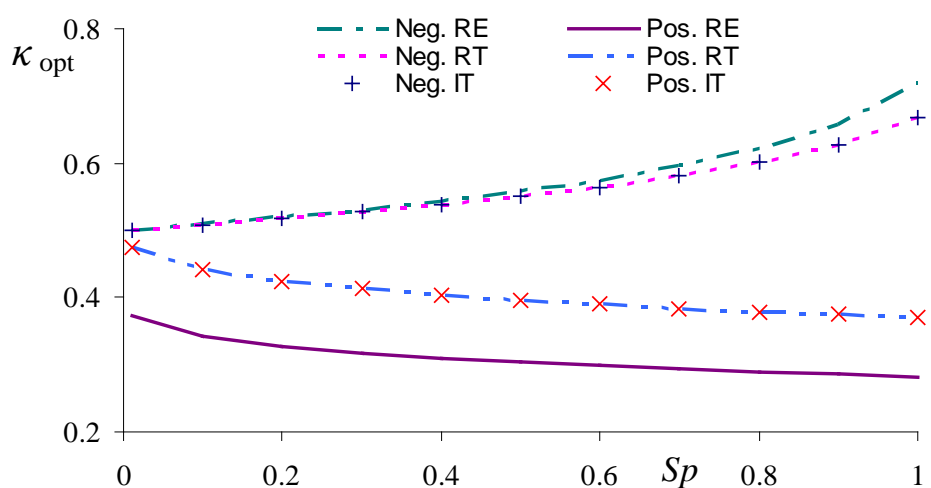

(b)

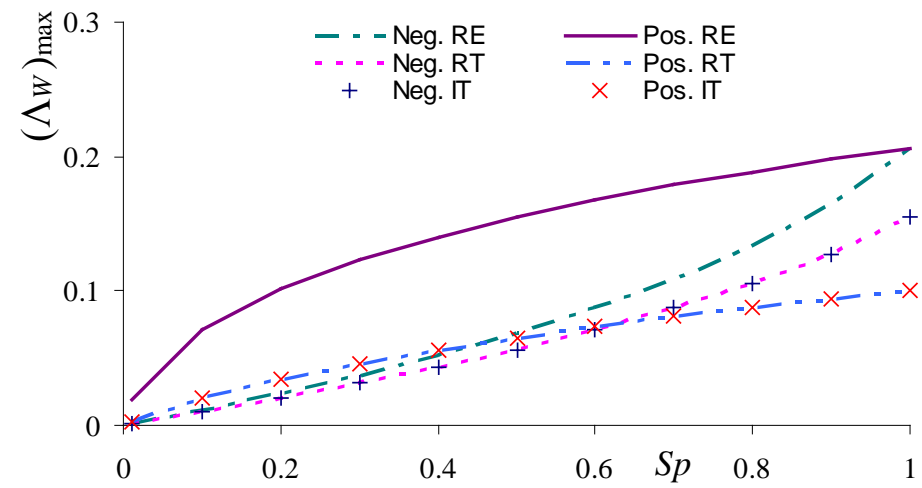

(c) 